\title{
Inhibition of Drp1 provides neuroprotection in vitro and in vivo
}

\author{
J Grohm ${ }^{1}$, S-W Kim ${ }^{2,3}$, U Mamrak ${ }^{2,3}$, S Tobaben ${ }^{1}$, A Cassidy-Stone ${ }^{4}$, J Nunnari ${ }^{4}$, N Plesnila ${ }^{2,3}$ and C Culmsee ${ }^{\star, 1}$
}

Impaired regulation of mitochondrial dynamics, which shifts the balance towards fission, is associated with neuronal death in age-related neurodegenerative diseases, such as Alzheimer's disease or Parkinson's disease. A role for mitochondrial dynamics in acute brain injury, however, has not been elucidated to date. Here, we investigated the role of dynamin-related protein 1 (Drp1), one of the key regulators of mitochondrial fission, in neuronal cell death induced by glutamate toxicity or oxygen-glucose deprivation (OGD) in vitro, and after ischemic brain damage in vivo. Drp1 siRNA and small molecule inhibitors of Drp1 prevented mitochondrial fission, loss of mitochondrial membrane potential (MMP), and cell death induced by glutamate or tBid overexpression in immortalized hippocampal HT-22 neuronal cells. Further, Drp1 inhibitors protected primary neurons against glutamate excitotoxicity and OGD, and reduced the infarct volume in a mouse model of transient focal ischemia. Our data indicate that Drp1 translocation and associated mitochondrial fission are key features preceding the loss of MMP and neuronal cell death. Thus, inhibition of Drp1 is proposed as an efficient strategy of neuroprotection against glutamate toxicity and OGD in vitro and ischemic brain damage in vivo.

Cell Death and Differentiation (2012) 19, 1446-1458; doi:10.1038/cdd.2012.18; published online 2 March 2012

Mitochondria play crucial roles in energy metabolism, regulation of free radical formation and calcium storage, thereby determining essential metabolic functions and cell survival. ${ }^{1}$ Further, mitochondria are highly dynamic organelles that undergo constant fission and fusion and these morphological changes are required for efficient ATP production, calcium buffering, regulation of signal transduction and apoptosis. ${ }^{2}$ In neurons, mitochondrial fission is also essential for axonal transport of the organelles into areas of high metabolic demand, ${ }^{3}$ whereas mitochondrial fusion supports substitution and regeneration of mitochondrial proteins, mtDNA repair and functional recovery. ${ }^{2,4}$

Consistent with the critical roles of mitochondrial dynamics in neurons, defects in mitochondrial fission and fusion proteins are associated with a wide array of inherited or acquired neurodegenerative diseases such as Charcot-Marie-Tooth disease or Alzheimer's disease, respectively. ${ }^{2}$ Fission and fusion defects may limit mitochondrial motility, decrease energy production, promote oxidative stress and lead to accumulating of mtDNA defects, thereby promoting neuronal dysfunction and cell death. ${ }^{1}$ Recently, enhanced mitochondrial fragmentation was associated with induction of neuronal death triggered by oxidative stress. ${ }^{5}$ These data imply that during neuronal cell death, the tubular mitochondrial network is fragmented into smaller and functionally impaired organelles. ${ }^{5}$ It is, however, a matter of ongoing controversy, whether mitochondrial fragmentation is cause or consequence in programmed cell death.

Current knowledge of the mechanisms regulating mitochondrial dynamics indicates that fission and fusion of mitochondria are under control of highly conserved dynamin-related GTPases. ${ }^{6}$ In mammalian cells, mitochondrial

\footnotetext{
${ }^{1}$ Institut für Pharmakologie und Klinische Pharmazie, Fachbereich Pharmazie, Philipps-Universität Marburg, Karl-von-Frisch-Straße 1, Marburg 35032, Germany; ${ }^{2}$ Department of Neurodegeneration, Royal College of Surgeons in Ireland (RCSI), Dublin, Ireland; ${ }^{3}$ Department of Physiology, Royal College of Surgeons in Ireland (RCSI), Dublin, Ireland and ${ }^{4}$ Department of Molecular and Cellular Biology, University of California, Davis, CA 95616, USA

*Corresponding author: C Culmsee, Institute for Pharmacology and Clinical Pharmacy, Faculty of Pharmacy, Philipps-University of Marburg, Karl-von-Frisch-Strasse 1, Marburg 35032, Germany. Tel: + 49642128 25780; Fax: + 49642128 25720; E-mail: culmsee@ @staff.uni-marburg.de

Keywords: neuronal cell death; oxidative stress; Drp1; mitochondrial fusion and fission; cerebral ischemia

Abbreviations: AIF, apoptosis-inducing factor; AD, Alzheimer disease; ATP, adenosinetriphosphate; Bcl-2, B-cell lymphom-2; Bak, Bcl-2 antagonist/killer; Bax, Bcl-2associated protein X; BCA, bicinchoninic acid; Bcl-xl, Bcl-2-related form log isoform; Bid, Bcl-2 interacting domain death antagonist; Bodipy, 4,4-diflouro-5-(4-phenyl1,3butadienyl)-4-bora-3a,4a-diaza-s-indacene-3-undecanoic acid; CCCP, carbonylcyanide-3-chlorophenylhydrazone; Cl, cell index; DAPI, 4',6-diamidino-2-phenylindole dihydrochloride; DMSO, dimethylsulfoxide; DMEM, Dulbecco's modified Eagle medium; DNA, deoxyribonucleic acid; Drp1, dynamin-related protein 1; EBSS, Earl's balanced salt solution; FACS, fluorescence activated cell sorting; FCS, fetal calf seum; GAPDH, glyceraldehyde-3-phosphate-dehydrogenase; GSH, gluthatione; h, hour; $\mathrm{H}_{2} \mathrm{O}_{2}$, hydrogen peroxide; HBSS, Hank's balanced medium; $\mathrm{HCl}$, hydrochloric acid; HEPES, 4-(2-hydroxyethyl)piperazine-1-ethanesulfonic acid; HRP, horseradish peroxidase; JC-1, 5, 5', 6, 6'-tetrachloro-1, 1' , 3,3'-tetraethylbenzimid-azolylcarbocyanine iodide; LDF, Laser-Doppler flowmetry; LCBF, local cortical blood flow; MABP, mean arterial blood pressure; MCAO, middle carotid artery occlusion; mdivi, mitochondrial division inhibitor; mGFP, mitochondrial targeting green fluorescent protein; MEM + , Eagle's minimum essential medium; Mfn-1, -2, mitofusin-1, -2; MMP, mitochondrial membrane potential; MTT, 3-(4,5-dimethylthiazol-2-yl)2, 5-diphenyltetrazolium bromide; MMP, mitochondrial membrane permeability; MOM, mitochondrial outer membrane; MOMP, mitochondrial outer membrane permeabilization; NMDA, $N$-methyl-D-aspartic acid; NCl, normalized cell index; OGD, oxygen-glucose deprivation; OPA1, optic atrophy 1; PBS, phosphate buffered saline; PCD, programmed cell death; PD, Parkinson's disease; PEI, polyethylenimine; PFA, paraformaldeyde; pH, potentia hydrogenii; PI, propidium iodide; PLL, poly-Lornithine; RT, room temperature; ROS, reactive oxygen species; SDS, sodium dodecyl sulfate; SDS-PAGE, sodium dodecyl sulfate polyacrylamide gel electrophoresis; S.D., standard deviation; S.E.M., standard deviation of the mean
}

Received 15.2.11; revised 23.1.12; accepted 31.1.12; Edited by N Chandel; published online 02.3.12 
membrane fusion is mediated by mitofusin-1 and -2 (Mfn-1, -2) and optical atrophy 1 (OPA1), respectively. ${ }^{6}$ Mitochondrial fission is mediated by dynamin-related protein 1 (Drp1). ${ }^{7-9}$ It has been suggested that the outer membrane proteins Fis1 and Mff and the proapoptotic Bcl-2 family protein $\mathrm{Bax}^{10,11}$ function directly or indirectly as Drp1 receptors to promote mitochondrial fission. The underlying mechanisms that promote and regulate the recruitment of cytosolic Drp1 to mitochondria are largely unknown.

The observation that enhanced mitochondrial fission is linked to neurodegeneration exposed Drp1 as a potential therapeutic target. Drp1 is highly conserved and contains many critical functional features that correspond to specific target structures within the enzyme, such as GTP binding, GTP hydrolysis, self-assembly and protein interactions with key functions in mitochondrial division. ${ }^{12,13}$

In the present study, the mechanisms of Drp1-dependent mitochondrial death pathways were investigated in a model of glutamate toxicity in immortalized mouse hippocampal HT-22 neurons (HT-22 cells). This cell line lacks ionotropic glutamate receptors and glutamate-induced death is mediated by inhibition of the cellular cystine import, subsequent glutathione depletion and enhanced lipoxygenation, inducing a form of programmed cell death called oxytosis. ${ }^{14-16}$ Further, we examined small molecule inhibitors of Drp $1^{17}$ to address whether Drp1 played a major role in neuronal cell death after glutamate toxicity and oxygen-glucose deprivation (OGD) in primary neurons in vitro and ischemic brain damage in vivo.

\section{Results}

siRNA silencing of Drp1 attenuates glutamate-induced mitochondrial fragmentation and cell death in HT-22 cells. Similar to our previous findings, glutamate toxicity in HT-22 cells was associated with mitochondrial fragmentation. ${ }^{5}$ Under control conditions, mitochondria were primarily organized in an evenly distributed tubular network, indicating a balance between mitochondrial fusion and fission events (Figures $1 \mathrm{a}$ and $\mathrm{b}$ ). After exposure to glutamate, mitochondria appeared as short tubules and round fragments (Figures $1 \mathrm{a}$ and b). Drp1 siRNA significantly reduced Drp1 mRNA and protein levels $>60 \%$ in the HT-22 cells (Figure 1e). Drp1 siRNA, but not control scrambled siRNA also attenuated mitochondrial fragmentation in glutamate-treated cultures (Figures $1 \mathrm{a}$ and $\mathrm{b}$; Supplementary Figures $1 \mathrm{a}$ and $\mathrm{b}$ ). In addition, silencing of Drp1 enhanced cell viability in glutamate-treated HT-22 cells as detected by the MTT (3-(4,5-dimethylthiazol-2-yl)-2,5diphenyltetrazolium bromide) assay (Figure 1c) and real-time impedance measurements (Figure 1d). FACS analysis of apoptotic cells after FITC-Annexin-V-staining confirmed the protective effect of Drp1 siRNA pool (Supplementary Figure 1c). These experiments were repeated with two additional different single siRNA sequences targeting Drp1 at concentrations of $40 \mathrm{nM}$, confirming the specificity of the protective effect against glutamate toxicity by Drp1 silencing (Supplementary Figures $2 a-f$ ). Overall, these results indicate that Drp1 is a suitable target of therapeutic strategies for neuroprotection against glutamate toxicity.
Novel pharmacological inhibitors of Drp1 provide protective effects in HT-22 cells. To further test the therapeutic potential of Drp1 inhibition, we applied the small molecule compounds mdiviA and mdiviB, which were previously established as Drp1 inhibitors in vitro. ${ }^{17}$ MdiviA and mdiviB blocked glutamate-induced mitochondrial fragmentation (Figure 2a). The Drp1 inhibitors increased the overall mitochondrial length and thus shifted the majority of cells into category 1 , suggesting reduced mitochondrial fragmentation and increased fusion events (Figure 2b; Supplementary Figures $3 \mathrm{c}$ and $\mathrm{e}$ ). It has been observed that Drp1 assembly and recruitment to mitochondria is stimulated during apoptosis. ${ }^{9,12}$ Consistently, glutamate caused an increase in Drp1 clusters associated with mitochondria (Figure 3a). In contrast, the majority of Drp1 remained diffusely distributed in the cytoplasm in cells treated with mdiviA or mdiviB despite the glutamate challenge. The mitochondria appeared as a tubular network in cells treated with mdiviA or mdiviB, contrasting to increased mitochondrial fragmentation in cells undergoing glutamate-induced death. In mitochondrial fractions obtained up to $10 \mathrm{~h}$ after onset of glutamate exposure, Drp1 immunoreactivity increased compared with controls in a time-dependent manner (Figure 3d; Supplementary Figure 4c). MdiviA blocked mitochondrial translocation of Drp1 (Figures $3 b$ and $c$ ). These results suggested that mdiviA and mdiviB attenuated mitochondrial division during glutamate-induced oxytosis by blocking Drp1 recruitment to mitochondria, consistent with previously published work on STS-induced cell death in mammalian COS-cells. ${ }^{12,17}$ In addition, mdiviA and mdiviB enhanced cell viability of glutamate-treated HT-22 cells as detected by impedance measurements (Figure 2c) and the MTT assay (Supplementary Figures $3 a$ and $d$ ). It is interesting to note that, once initiated, the complete loss of cellular impedance was observed within a relatively small time window of $2-3 \mathrm{~h}$, suggesting that cell death occurred in a highly synchronized manner, and this was persistently blocked by the Drp1 inhibitors (Figure 2). These results were also confirmed by FACS analysis after FITC-Annexin-V-staining (Figure 2d; Supplementary Figure $3 b$ ). In addition, mdiviA also prevented ATP depletion (Figure 3e; Supplementary Figures $3 f$ and $g$ ) and significantly reduced lipid peroxidation after glutamate exposure (Figure 3f). In this model system, the increase in early ROS formation after $6-8 \mathrm{~h}$ through increased lipid peroxidation is followed by a second more pronounced accumulation of ROS caused by damage of mitochondria. ${ }^{16}$ Here, lipid peroxidation was detected at the later stages of cell damage, when mitochondrial ROS formation occurred in glutamate-damaged cells. This late increase in ROS formation was blocked by the Drp1 inhibitor mdiviA, indicating protection against mitochondrial ROS formation (Figure 3f). Analyses of mitochondrial protein extracts at various time points after onset of the glutamate treatment revealed increased Drp1 translocation to the organelles at 8-10 h of glutamate exposure (Figure 3d). The Drp1 translocation to mitochondria was confirmed by immunostainings of Drp1 staining (Supplementary Figure 4a) and matches the time course of cellular death that also became detectable around $8 \mathrm{~h}$ by impedance measurements 
a
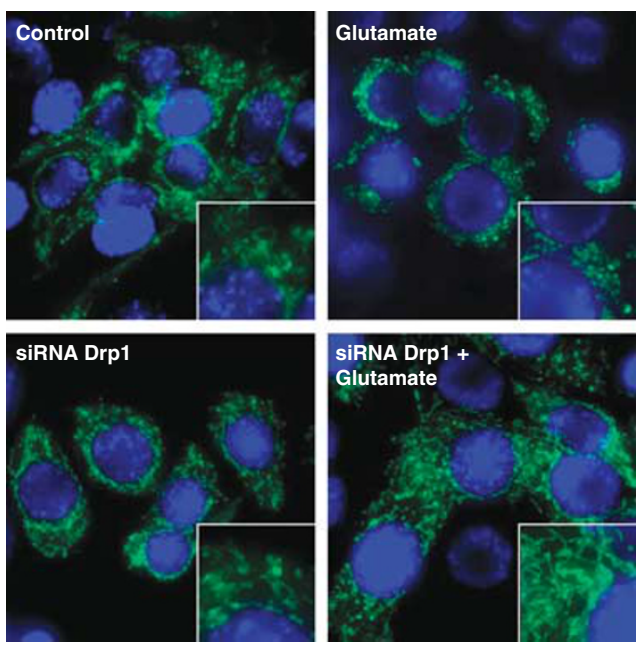

b $\left.{ }^{100}\right] \square$ Category 1

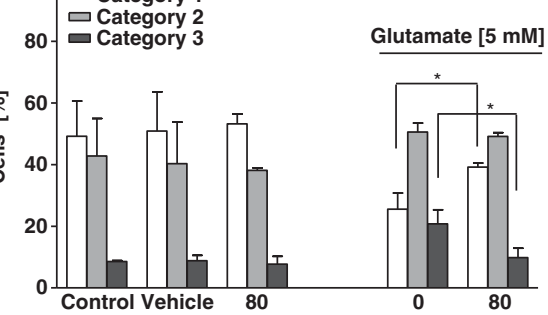

C $200 \square$ Control siRNA Drp1 [nM]

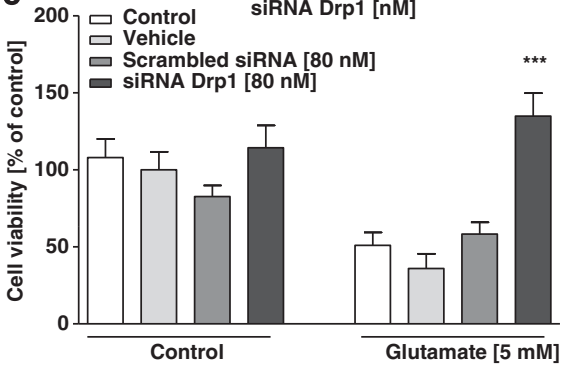

d 4 to- Control Vehicle

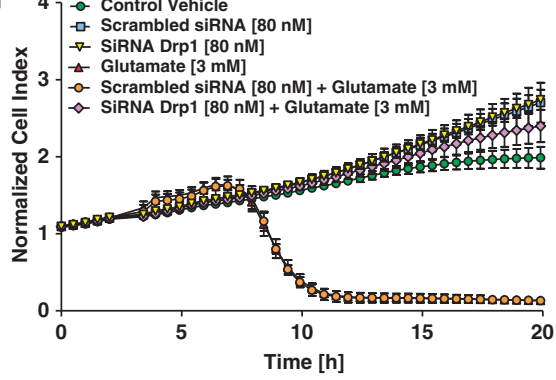

e

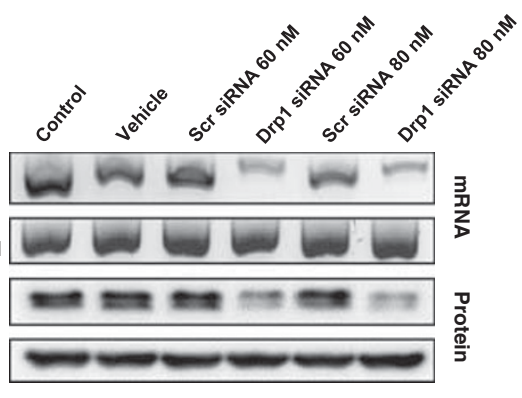

Figure 1 siRNA silencing of Drp1 attenuates glutamate-induced mitochondrial fragmentation and cell death in HT-22 cells. (a) Fluorescence photomicrographs $(\times 63$ objective) of mGFP-transfected and DAPI-stained HT-22 cells show that pooled Drp1 siRNA ( $80 \mathrm{nM}, 48 \mathrm{~h}$ ) prevents fission of mitochondria in glutamate-exposed ( $5 \mathrm{mM}, 18 \mathrm{~h}$ ) HT-22 cells. (b) Quantification of mitochondrial morphology of $\sim 500$ cells per condition: category 1 (elongated tubulin-like structure), category 2 (intermediate length) and category 3 (fragmented mitochondria). ${ }^{*} P<0.05$ compared with glutamate $5 \mathrm{mM}$-treated cells $(n=3$ independent experiments, ANOVA, Bonferroni-test). (c) Silencing of Drp1 attenuates glutamate-induced cell death as determined by MTT assay. The presented data are normalized to vehicle control Lipofectamine $2000(100 \%)$. ${ }^{* \star *} P<0.001$ compared with glutamate-treated cells ( $n=8$, ANOVA, Scheffe's-test). For statistical analysis, the experiments were independently repeated at least three times with an $n=8$ per treatment condition and the results are presented as mean \pm S.D. (d) Representative graph of real-time detection of cellular impedance by $x C E L L i g e n c e ~ S y s t e m$ (Roche) is shown. After $24 \mathrm{~h}$, Drp1 siRNA-transfected HT-22 cells were seeded out for additional $24 \mathrm{~h}$ in 96-well E-plates with 4500 cells/well and treated with $3 \mathrm{mM}$ glutamate for $20 \mathrm{~h}$ ( $n=8$ per treatment condition). Glutamate-induced cell death does not differ in vehicle- or scrambled siRNA-treated cells, whereas Drp1 siRNA exerts a persistent protective effect. The cellular impedance of glutamate alone (red triangles) is obscured by the overlapping curve of scrambled siRNA + glutamate (orange dots). (e) RT-PCR analysis of Drp1 mRNA (upper panels) and western blot analysis of Drp1 protein (lower panels) in HT-22 cells pretreated with 60 and $80 \mathrm{nM}$ pooled Drp1 siRNA for $48 \mathrm{~h}$. RT-PCR with primers specific for glyceraldehyde-3-phosphate-dehydrogenase (GAPDH) and anti- $\beta$-actin antibodies used as controls for respective analyses is shown. Quantification of the relative protein expression shows a reduction of 60 and $70 \%$

or MTT assays (Figure 1d). These observations indicated a continuous increase in Drp1 translocation to mitochondria while the organelles were fragmented and accumulated around the nucleus in damaged cells. Quantification of cells with Drp1 translocation to the mitochondria (Supplementary Figure 4b) and additional western blot analysis with protein quantifications determined Drp1 translocation to the mitochondria after $10 \mathrm{~h}$ of glutamate toxicity in HT-22 cells, which was blocked by mdiviA (Figures $3 b-d)$ ). Overall, these results reveal a major upstream role for Drp1 in mitochondrial damage, energy depletion and subsequent execution of glutamate-induced cell death in HT-22 cells.

It is important to note that the non-functional mdiviA derivate mdiviG $^{17}$ also failed to inhibit glutamate-induced mitochondrial fragmentation and cell death in the HT-22 cells (Supplementary Figures $5 \mathrm{a}-\mathrm{c}$ ). In contrast, the active mdiviA derivatives mdiviC and mdiviE preserved mitochondrial morphology and cell viability with similar effectivity as mdiviA (Supplementary Figures $5 d$ and e). To further verify the proposed specificity of mdivi inhibitors, we combined treatment with Drp1 siRNA and mdiviA. The combination of Drp1 siRNA and mdiviA did not exceed the protective effects of the respective treatments alone (Supplementary Figure 6a). Notably, the combination of both approaches appeared to decrease the therapeutic effect rather than exerting additional protection, consistent with toxic effects of mdivi compounds at high concentrations and cytotoxic effects of dominant negative mutants of Drp1 ${ }^{18}$ Next, we determined the 
a
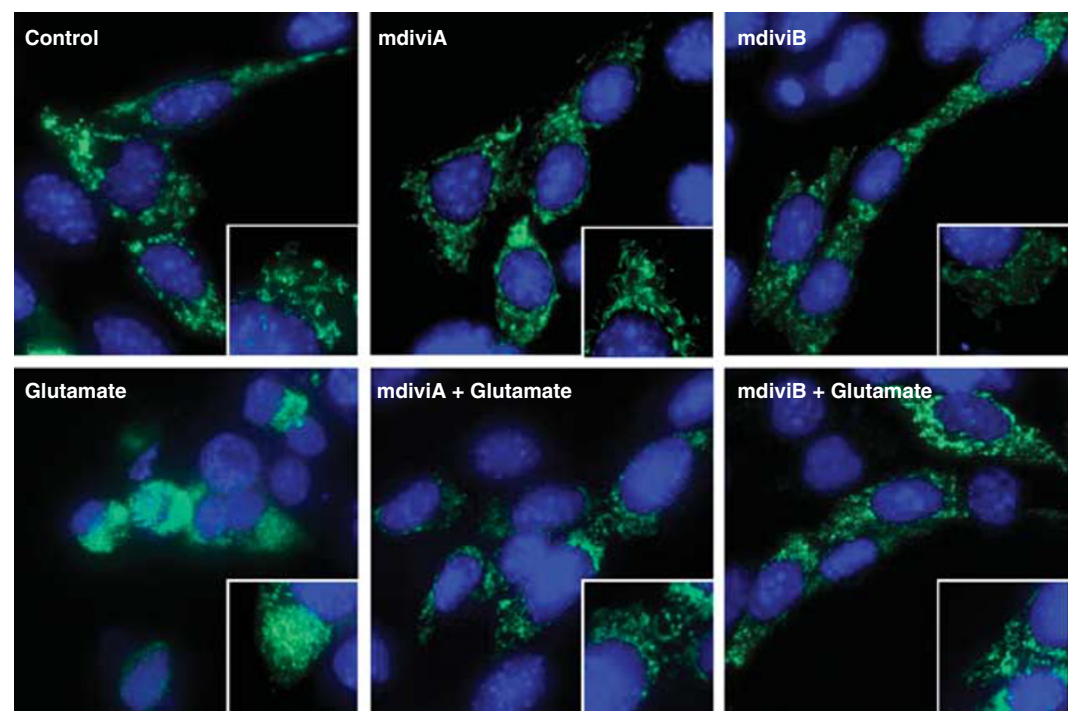
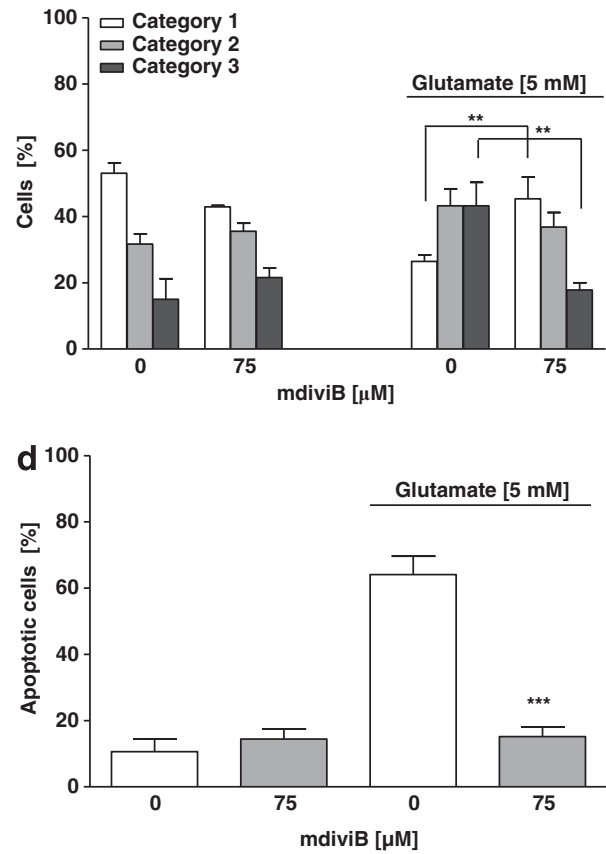

Figure 2 The novel pharmacological inhibitor of Drp1, mdiviA, protects HT-22 cells against glutamate toxicity. (a) Fluorescence photomicrographs ( $\times 63$ objective) of mGFP-transfected and DAPI-stained HT-22 cells show that Drp1 inhibitor mdiviA (75 $\mu \mathrm{M})$ prevents fission of mitochondria in glutamate-exposed $(5 \mathrm{mM}, 18 \mathrm{~h}) \mathrm{HT}-22$ cells. (b) Quantification of mitochondrial morphology of $\sim 500$ cells per condition: category 1 (elongated tubulin-like structure), category 2 (intermediate length) and category 3 (fragmented mitochondria). ${ }^{* \star} P<0.01$ category $1+2$ mdiviA, mdiviB + glutamate compared with glutamate $5 \mathrm{mM}$-treated cells $(n=3$ independent experiments, ANOVA, Bonferroni-test). (c) HT-22 cells were cultured for $48 \mathrm{~h}$ in 96-well E-plates with 4500 cells/well and treated $24 \mathrm{~h}$ after seeding with 3 and $5 \mathrm{mM}$ glutamate and mdiviA (75 $\mu \mathrm{M}$ ) for $18 \mathrm{~h}(n=8)$. Representative graph of real-time detection of cellular impedance by xCELLigence System (Roche) is shown. (d) FACS analysis of HT-22 cells with $n=10000$ cells per treatment condition after FITC-Annexin-V labeling to detect apoptotic cells. Exposure to glutamate $(5 \mathrm{mM}, 14 \mathrm{~h})$ results in enhanced Annexin-V binding of apoptotic HT-22 cells compared with DMSO control. MdiviB $(75 \mu \mathrm{M})$ significantly reduce glutamate-induced apoptosis. For statistical analysis, the experiments were repeated at least three times with an $n=3$ per treatment condition. ${ }^{\star \star \star} P<0.001$ compared with glutamate-treated cells ( $n=3$, ANOVA, Scheffé's-test)

protective time window and added the Drp1 inhibitors mdiviA, $B$ and $G$ at different time points between 2 and $12 \mathrm{~h}$ after onset of the glutamate treatment. HT-22 cells were protected against glutamate toxicity even when mdiviA or mdiviB were added up to $8 \mathrm{~h}$ after the glutamate challenge (Supplementary Figure 6b). MdiviG, the non-functional mdivi compound failed to protect the cells in the post-treatment experiments (Supplementary Figure 6b).

Drp1 gene silencing and pharmacological Drp1 inhibition prevent mitochondrial depolarization and Drp1 translocation to the mitochondria. Loss of 

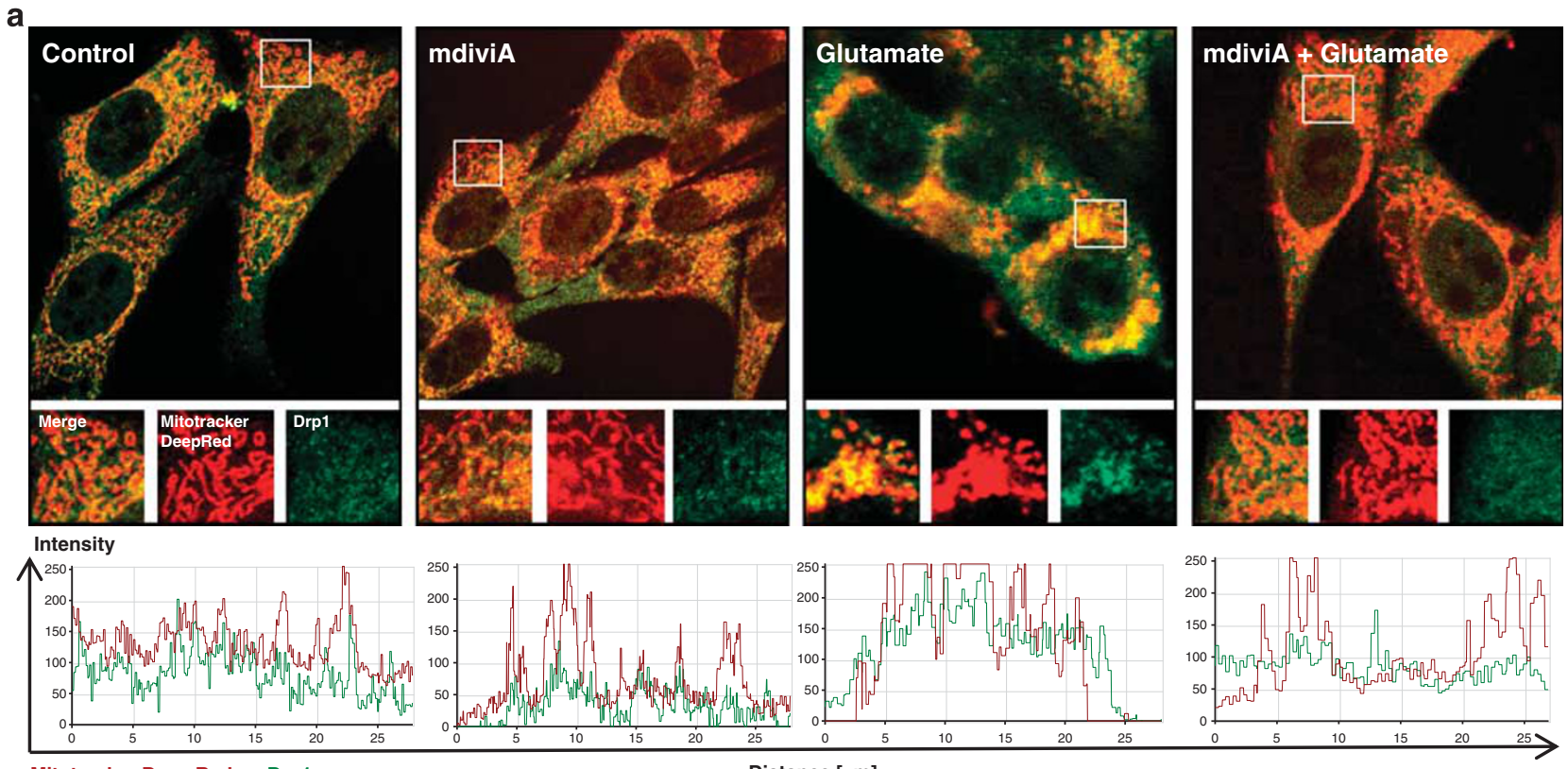

Mitotracker Deep Red Drp1

b

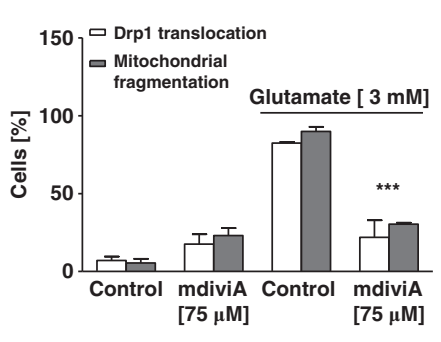

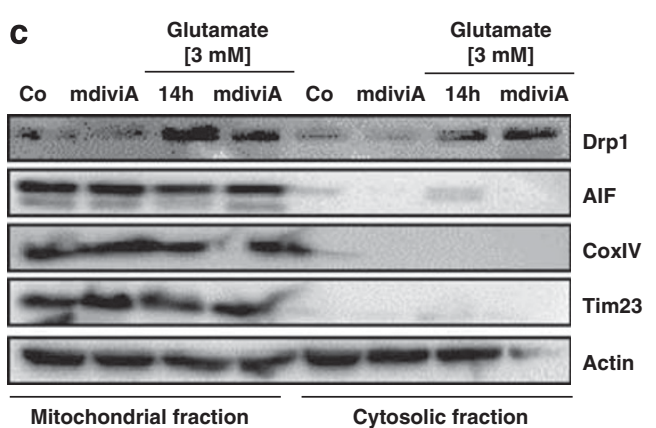

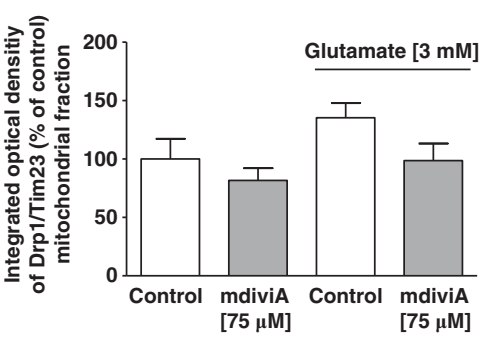

d $\frac{\text { Glutamate }[3 \mathrm{mM}]}{8 \mathrm{~h} 10 \mathrm{~h} 13 \mathrm{~h} 15 \mathrm{~h}}$ co $\frac{\text { Glutamate }[3 \mathrm{mM}]}{8 \mathrm{~h} 10 \mathrm{~h} 13 \mathrm{~h} 15 \mathrm{~h}}$
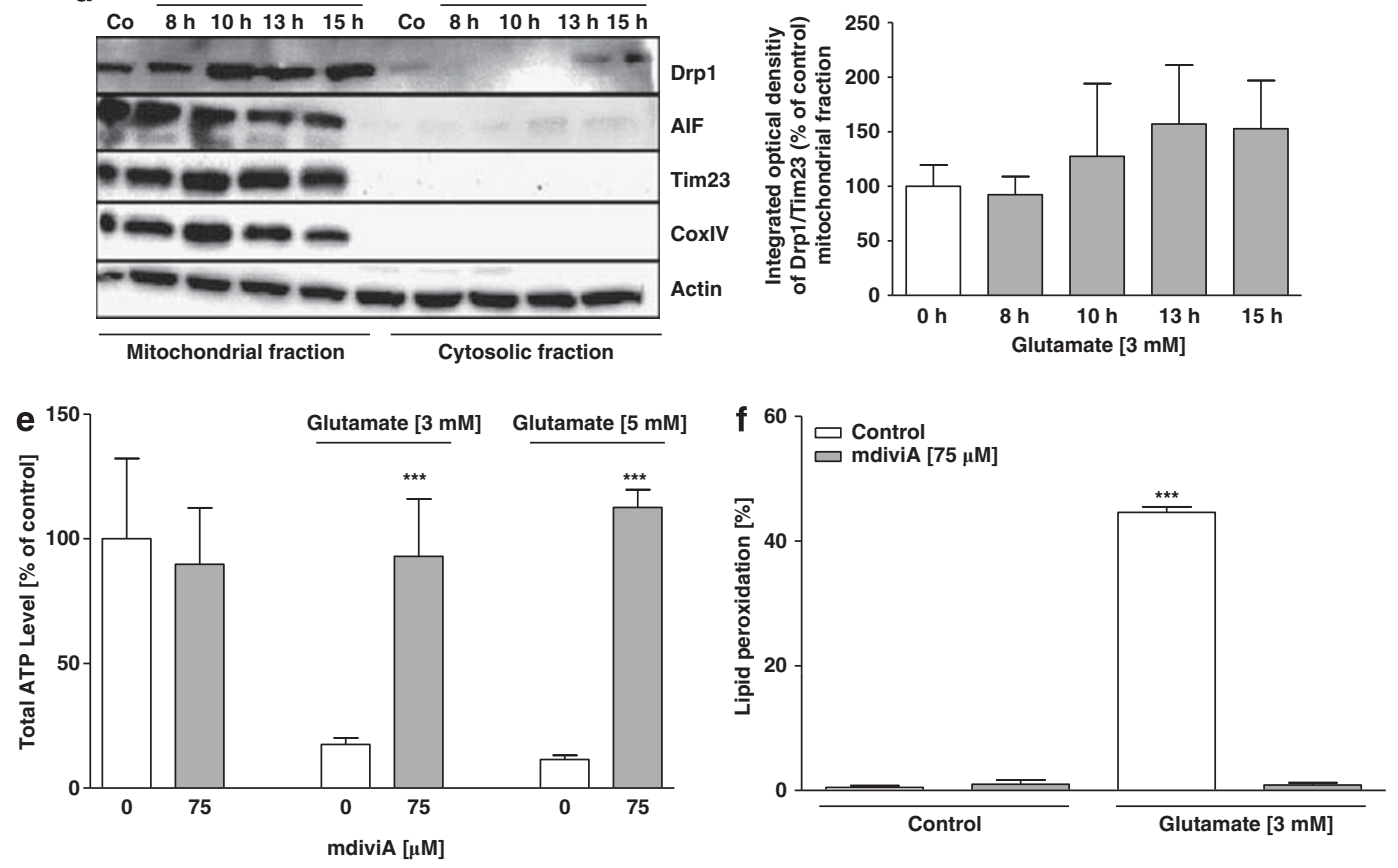
mitochondrial membrane potential (MMP) is a major feature of glutamate-induced cell death that is associated with mitochondrial fragmentation and precedes the fatal release of AIF and execution of caspase-independent cell death. ${ }^{5,19}$ In line with our previous experiments in HT-22 cells, JC-1 fluorescence indicated that there was a decrease in MMP within $12 \mathrm{~h}$ after glutamate treatment, similar to that observed after treatment with the uncoupler carbonylcyanide-3chlorophenylhydrazone (CCCP) (Figures 4a and c). Three different Drp1 siRNAs significantly attenuated the glutamateinduced breakdown of the MMP, and similar effects were detected after using the Drp1 inhibitors mdiviA and mdiviB (Figures 4b and d; Supplementary Figure 7).

Inhibition of Drp1 prevents tBid-induced mitochondrial fragmentation and neuronal cell death. Our recent data demonstrated a role for the proapoptotic $\mathrm{BH} 3$-only protein Bid for enhanced mitochondrial fragmentation in the present model systems of HT-22 cell oxytosis and primary neuron cell death induced by glutamate or OGD. ${ }^{5,19}$ We documented that Bid is involved in the loss of MMP and release of mitochondrial AIF, which mediates delayed neuronal death after cerebral ischemia. ${ }^{19-21}$ Here, we examined the role of Drp1 in Bid-mediated neuronal cell death and observed that treatment of cells with mdiviA or mdiviB also prevented tBid-induced mitochondrial fission and preserved cell viability (Figure 5). Vector-induced expression of tBid reduced cell viability to $30 \%$ of control levels, whereas cell viability was restored to control levels when the Drp1 inhibitors were applied to tBid expressing cells (Figure $5 \mathrm{c}$ ). This protective effect of mdiviA and $B$ against tBid-induced cell death was confirmed in FACS analysis of apoptotic cells after FITC-Annexin-V-staining (Figure 5d).

It is known that full-length Bid as well as cleaved Bid translocating to mitochondria in dying cells contributes to mitochondrial demise in HT-22 cells and may explain why overexpression of tBid triggers very similar Drp1-dependent mitochondrial death pathways. ${ }^{22,23}$ Additional experiments using Bid siRNA to further substantiated the role of Bid in Drp1-mediated mitochondrial fission (Supplementary Figures 8a-e). As shown in Supplementary Figure 8, silencing of Bid prevents mitochondrial fragmentation and the loss of cell viability. Further, silencing of Bid inhibits the translocation of Drp1 caused by glutamate and clearly demonstrates that Bid plays a significant role in Drp1-dependent mitochondrial fission. Since Bid siRNA inhibit both, full-length Bid and tBid toxicity, similar results are detected in native cells and in cells overexpressing tBid. A direct interaction between Bid/tBid and Drp1, however, could not be detected in immunoprecipitation experiments (Supplementary Figure 9).

Mdivi compounds protect primary neurons from glutamate toxicity and OGD in vitro and attenuate ischemic brain damage in vivo. Because HT-22 cells lack i receptors, the HT-22 model of oxytosis only partly reflects the mechanisms of neuronal death after acute brain injury. Thus, we next addressed the effects of the mdivi compounds in a model of glutamate-induced excitotoxicity in primary cortical neurons, which express these ionotropic glutamate receptors. We found that mdivi A or B significantly attenuated OGD-induced damage in the primary neurons (Figures $6 \mathrm{a}$ and $\mathrm{b}$ ), and similar protective effects were detected in primary neurons exposed to glutamate (Figure 6c). Control neurons displayed condensed, pycnotic nuclei $18 \mathrm{~h}$ after OGD, whereas the neurons receiving mdivi compounds maintained healthy nuclear morphology (Figure 6a). Quantification of four independent experiments revealed that mdivi compounds $A$ or B reduced OGDinduced cell death by $\sim 55 \%$ (Figure $6 \mathrm{~b}$ ).

Findings on the efficacy of mdivi compounds in the in vitro models encouraged further investigations in vivo, in a model of ischemic stroke (Figure 6d). Remarkably, mdiviA (3 mg/kg) or $\mathrm{mdiviB}$ ( 1 and $3 \mathrm{mg} / \mathrm{kg}$ ) reduced the mean infarct volume by $34 \%$ and $30 \%$ compared with vehicle (dimethylsulfoxide (DMSO)) controls, respectively. These data demonstrate a significant protective efficiency of the mdivi compounds in vivo and suggested that the mechanisms of Drp1-mediated mitochondrial pathways of neuronal cell death also play a major role in ischemic brain damage. Notably, analysis of physiological parameters did not reveal any differences between mdiviA-treated animals and vehicle controls. This observation was also confirmed in a toxicity study over 7 days,

Figure 3 MdiviA inhibits glutamate-induced translocation of Drp1 to the mitochondria, and prevents energy depletion and lipid peroxidation. (a) HT-22 cells were cultured for $24 \mathrm{~h}$ in eight-well-ibidi-plates and treated with $3 \mathrm{mM}$ glutamate and mdiviA $(75 \mu \mathrm{M})$ for $14 \mathrm{~h}$. Immunostaining with specific antibody of Drp1 reveal that Drp1 (green) translocation to the mitochondria (Mitotracker Deep Red) occur after glutamate stimulus. In the magnification pictures by overlapping of the fluorescence (yellow) is shown that Drp1 interacts with the mitochondria. MdiviA inhibits the translocation of Drp1 to the mitochondria. Analysis of fluorescence intensities by Zeiss image software revealed the co-localization of Drp1 and mitochondria by overlapping of green and red fluorescence. (b) Quantification of cells with localization of Drp1 at mitochondria. Drp1 translocation (white bar) correlates with increased mitochondrial fragmentation (grey bar) after glutamate $(3 \mathrm{mM}$ ) treatment. MdiviA inhibits the translocation of Drp1 to the mitochondria. The quantification is based on three independent experiments with an $n=60-70$ cells per treatment condition, which were analyzed without knowledge of the treatment history. ${ }^{* \star} P<0.001$ compared with glutamate ( 3 and $5 \mathrm{mM}$ )treated cells ( $n=3$, ANOVA, Scheffé's-test). (c) Representative western blot analysis of mitochondrial and cytosolic fractions of HT-22 cells show that glutamate ( $3 \mathrm{mM}$ ) induces an increase of endogenous Drp1 protein in the mitochondrial fraction after $14 \mathrm{~h}$. Treatment with mdiviA inhibit Drp1 translocation to the mitochondria. Quantitative analysis of protein amount was performed by intensity measurements of Drp1 in contrast to mitochondrial loading control Tim23. (d) Representative western blot analysis of mitochondrial and cytosolic fractions of HT-22 cells to detect the translocation of Drp1 to mitochondria after glutamate treatment in a time-dependent manner $(3 \mathrm{mM}, 8-15 \mathrm{~h})$. Glutamate-treated cells in comparison with the control cells (Tim23 for mitochondria protein loading; actin for cytosolic extracts). These experiments were independently repeated three times with similar results. The representative quantification of total Drp1 in relation to the respective loading controls in the cytosolic and mitochondrial fractions confirmed that Drp1 accumulates in the glutamate-treated cells in comparison with the control cells (Tim23 for mitochondria protein loading; actin for cytosolic extracts). These experiments were independently repeated three times with similar results. (e) HT-22 cells were cultured in white 96 -well plates with 7000 cells/well and treated with 3 and $5 \mathrm{mM}$ glutamate and mdiviA $(75 \mu \mathrm{M})$ at $14 \mathrm{~h}$. ATP-luminescence measurements were performed according to the manufacturer's protocol. The presented data are normalized to DMSO control. For statistical analysis, the experiments were repeated at least three times with an $n=8$ per treatment condition. ${ }^{* * *} P<0.001$ compared with glutamate ( 3 and 5 mM)-treated cells (ANOVA, Scheffe's-test). (f) Fluorescence detection of lipid peroxidation by Bodipy assay, quantified by FACS analysis of three independent experiments with $n=20000$ cells per treatment condition. ${ }^{* \star *} P<0.001$ compared with glutamate ( 3 and $\left.5 \mathrm{mM}\right)$-treated cells $(n=3$, ANOVA, Scheffé's-test) 
a

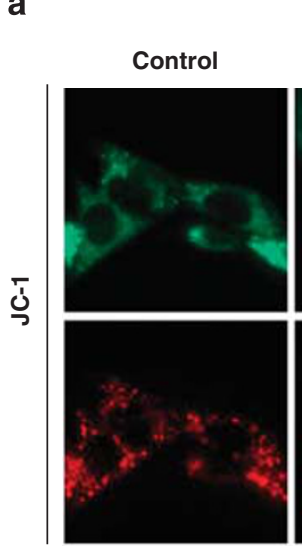

c

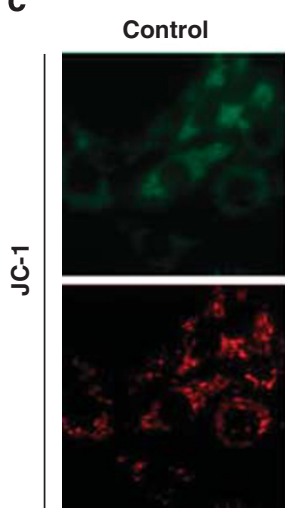

Glutamate
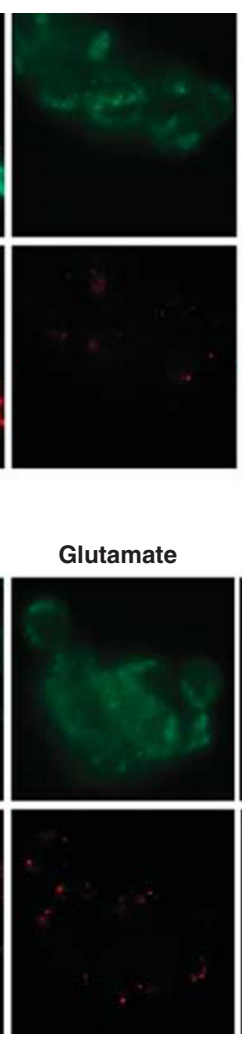

\section{siDrp1+} Glutamate

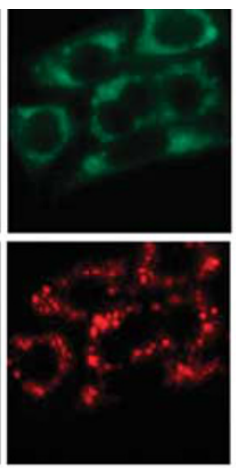

mdiviA + Glutamate

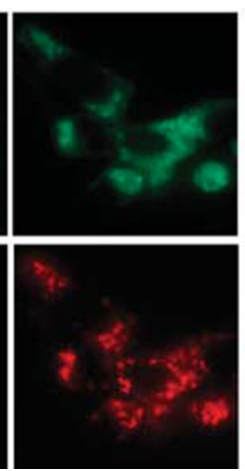

Scrambled siRNA+ Glutamate

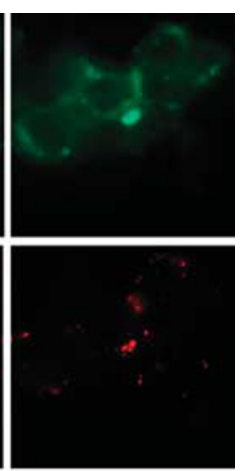

mdivi B+ Glutamate

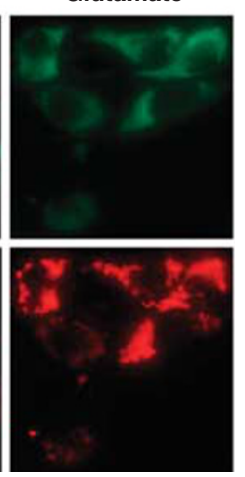

b

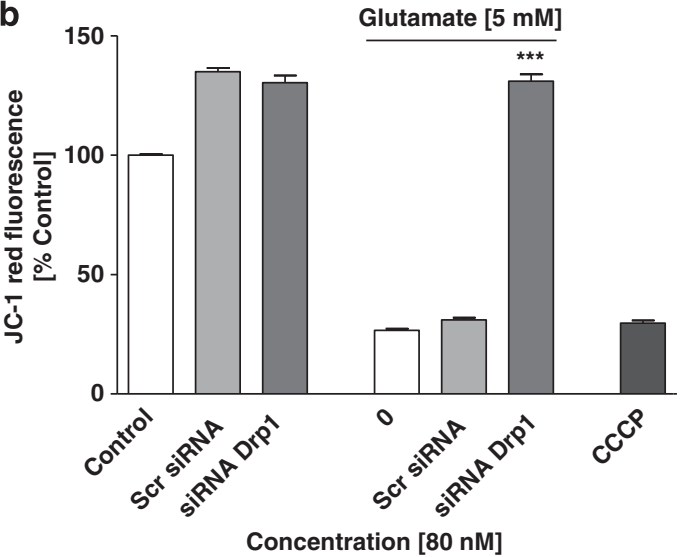

d

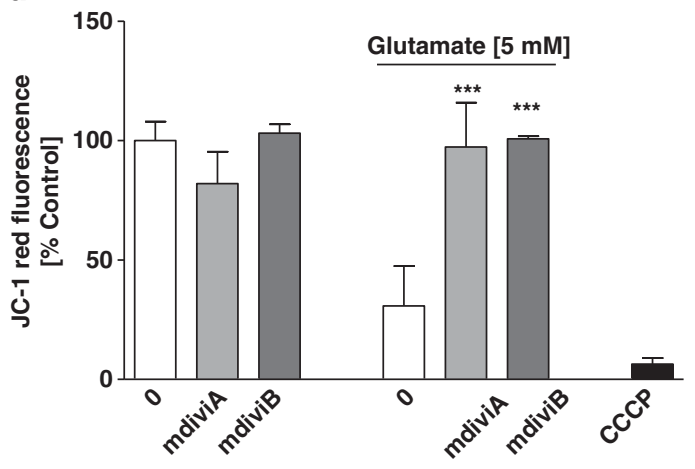

Concentration $[75 \mu \mathrm{M}]$

Figure 4 Inhibition of Drp1 prevents glutamate-induced mitochondrial depolarization. (a and c) MMP was analyzed by JC-1 fluorescence: upper panels show epifluorescence photomicrographs indicating equal cellular uptake of $\mathrm{JC}-1$ by green fluorescence; lower panels depict intact mitochondria exposing red fluorescence. Glutamate-treated $(5 \mathrm{mM}, 12 \mathrm{~h}) \mathrm{HT}-22$ cells show significantly reduce red fluorescence compared with controls whereas mdiviA and B (75 $\mu \mathrm{M})$ and siRNA of Drp1 ( $80 \mathrm{nM})$ prevents the breakdown of the MMP as indicated by preservation of the red JC- 1 fluorescence. (b and d) FACS analyses of $n=3$ independent experiments with 10000 cells per treatment condition reveal a decrease of the red JC- 1 fluorescence to $30 \%$ of control levels $12 \mathrm{~h}$ after glutamate treatment $(5 \mathrm{mM})$, which has prevented $\mathrm{mdiviA}$, B and siRNA of Drp1 $(80 \mathrm{nM})$. Glutamate treatment was as effective as the positive damage-control CCCP, which causes a fast breakdown of the MMP. The presented data are normalized to DMSO control for mdiviA and mdiviB and vehicle control Lipofectamine 2000 for siRNA approach (100\%). ${ }^{* *} P<0.001$ compared with glutamate-treated controls ( $n=3$, ANOVA, Scheffé's-test)

where temperature and body weight, blood gases, $\mathrm{pH}, \mathrm{Na}^{+}$ and $\mathrm{K}^{+}$-concentrations, and cerebral blood flow did not differ between the animals of the different groups (Supplementary Figure 10).

\section{Discussion}

The present study demonstrates that Drp1-mediated mitochondrial fission plays a major role in neuronal cell death associated with acute ischemic brain damage. This conclusion is based on effects of Drp1 siRNA or the small molecule inhibitors, which significantly preserved mitochondrial morphology and MMP, and reduced glutamate toxicity in the neuronal HT-22 cell line. Further, Drp1 inhibitors prevented glutamate excitotoxicity and OGD-induced death in primary cultured neurons, and reduced the infarct size in a model of cerebral ischemia in vivo. Therefore, the current findings extend previous reports on the role of Drp1 in chronic neurodegenerative diseases, since we demonstrate that Drp1 mediates neuronal death in models of acute brain injury. In fact, Drp1-mediated detrimental fission of mitochondria appears occurring independent of the initiating trigger mechanism. The model systems applied here differ regarding the initiation of cell damage, that is, glutamate-induced ROS formation in HT-22 cells, glutamate-induced excitotoxicity in primary neurons, OGD, glutamate excitotoxicity with a burst of ROS formation upon re-oxygenation in the model of OGD in primary neurons and after ischemic brain damage and reperfusion in vivo. All these models were suitable to reveal protective effects of Drp1 inhibition. Thus, regardless of the different upstream stress stimulus, Drp1 translocation and Drp1-mediated mitochondrial fragmentation and downstream mitochondrial death pathways play a major role for the observed cell death.

Our present findings demonstrating protective effects of Drp1 inhibitors in models of OGD in vitro and cerebral ischemia in vivo are in line with recent reports in experimental models of ischemia in the retina, the heart or the kidney. ${ }^{24-26}$ In addition, mdiviA was efficacious in rodent models of cisplatin-induced renal damage, ${ }^{24}$ suggesting a therapeutic potential for Drp1 inhibitors in tissue damage caused by different insults. Earlier studies using dominant negative 
a
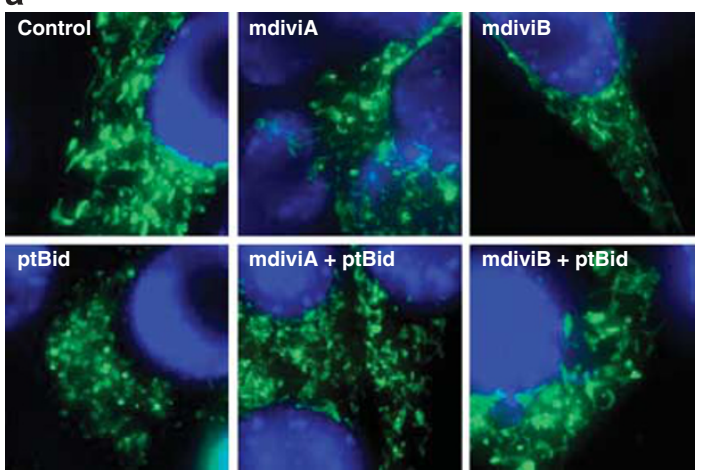

b

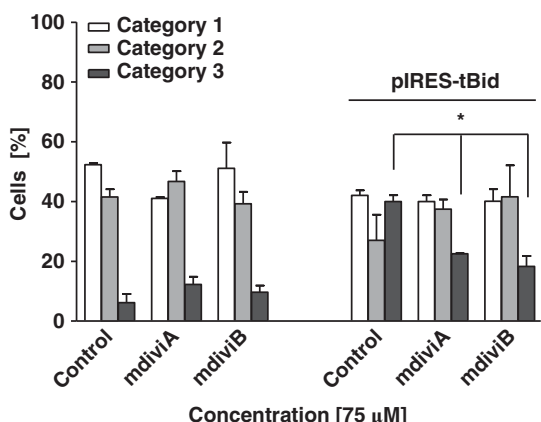

C

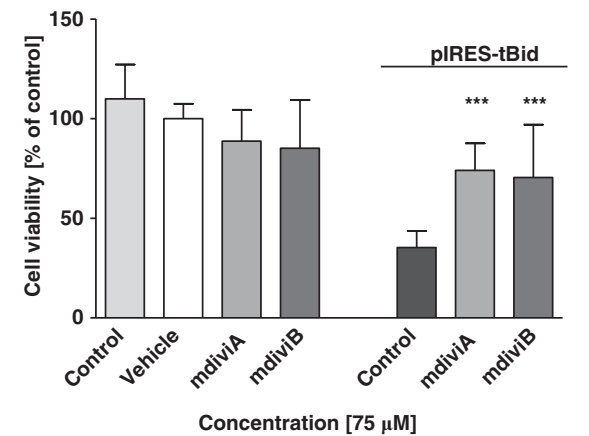

d 60 ○ Control

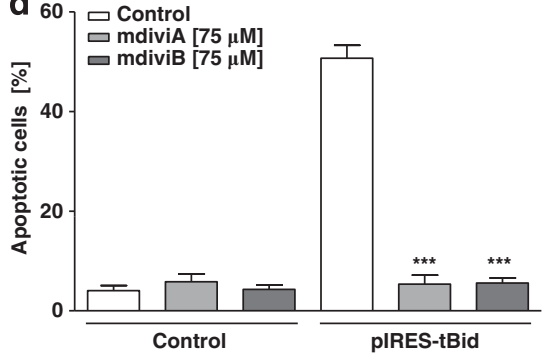

Figure 5 Inhibition of Drp1 prevents tBid-induced mitochondrial fragmentation and neuronal cell death. (a) Fluorescence photomicrographs $(\times 63$ objective) showed that the Drp1 inhibitors mdiviA and mdiviB $(75 \mu \mathrm{M})$ prevent fission of mitochondria in tBid-induced toxicity after $12 \mathrm{~h}$ in HT-22 cells. (b) Quantification of mitochondrial morphology of $\sim 500$ cells per condition: category 1 (elongated tubulin-like structure), category 2 (intermediate length) and category 3 (fragmented mitochondria). ${ }^{\star} P<0.05$ compared with glutamate $(5 \mathrm{mM})$-treated cells $(n=3$ independent experiments, ANOVA, Bonferroni-test). (c) HT-22 cells were cultured in 24 -well plates with 70000 cells/well and tBid-induced cell death determined by MTT assay after $12 \mathrm{~h}$. Treatment with mdiviA and mdiviB $(75 \mu \mathrm{M})$ was performed $1 \mathrm{~h}$ before transfection. The presented data are normalized to vehicle control Lipofectamine 2000 . ${ }^{* \star} P<0.001$, compared with tBid-treated cells ( $n=4$, ANOVA, Scheffé's-test). For statistical analysis, the experiments were independently repeated at least three times with an $n=4$ per treatment condition and the results are presented as mean \pm S.D. (d) FACS analysis of HT-22 cells in $n=3$ independent experiments with 10000 cells per treatment condition after FITC-Annexin-V labeling to detect apoptotic cells. Transfection of pIRES-tBid results in enhanced Annexin-V binding of apoptotic HT-22 cells compared with vehicle control Lipofectamine 2000 . MdiviA and $\mathrm{B}(75 \mu \mathrm{M})$ significantly reduce tBid-induced cell death. ${ }^{* \star *} P<0.001$ compared with tBid toxicity ( $n=3$, ANOVA, Scheffe's-test)

mutant Drp1K38A validated Drp1 as a potential therapeutic target in neurodegenerative diseases. ${ }^{7}$ In addition, a very recent study showed that enhanced Drp1 activity caused detrimental mitochondrial fission in Huntington's disease. ${ }^{27}$ This study also applied mdiviA, and together with our current findings it is suggested that Drp1 inhibition is a promising approach to prevent mitochondrial fragmentation in different models of delayed neuronal cell death relevant for acute and chronic neurological diseases. In fact, these studies show that Drp1 inhibitors are applicable to neurons in vitro and in vivo, thereby overcoming obstacles of siRNA applications or gene therapy. ${ }^{13}$

The trigger for Drp1 activation in delayed neuronal cell death has not yet been revealed, but there is growing evidence that Bcl-2 family proteins like Bax, Bak and Bid affect mitochondrial morphology and localization in healthy cells. ${ }^{10,28,29}$ For example, Bax can promote Drp1-dependent mitochondrial fragmentation by co-localization of Bax with Drp1 and Mfn-2 at sites of the mitochondria outer membrane (MOM), which then become fission sites. These protein interactions stabilize the association of Drp1 with the mitochondrial membrane and are prerequisite for inducing fragmentation of the organelles. ${ }^{28}$ In turn, Drp1 stimulates Bax oligomerization by accelerating formation of hemifusion/ fission structures of the mitochondrial membrane. ${ }^{11}$ Such Drp1 facilitated Bax oligomerization is likely an essential step towards enhanced membrane permeability, and the Baxdependent release of proapoptotic factors from the mitochondria into the cytosol. Further, Bax translocation to the mitochondria correlates with a reduction of mitochondrial fusion since Bax inhibits mitofusins thereby decreasing mitochondrial fusion, ${ }^{11}$ and this Bax activity may be facilitated by activated Bid or tBid. ${ }^{20}$

Our recent data demonstrate a pivotal role for the proapoptotic $\mathrm{BH} 3-o n l y$ protein Bid in the glutamate-induced oxidative stress model system. Further, depolarization of the MOM and mitochondrial fragmentation are both important key events of apoptosis induced by oxidative stress, and both events were apparently Bid dependent (Supplementary Figures $8 a-d) .{ }^{5,19}$ The pivotal role for Bid upstream of such mitochondrial dysfunction is substantiated by experiments demonstrating pronounced neuroprotective effects of the Bid inhibitor $\mathrm{BI}-6 \mathrm{c} 9$ in models of glutamate-induced excitotoxicity and OGD in primary cultured neurons, and similar studies in HT-22 neurons using Bid siRNA. ${ }^{19,20,30,31}$ Further, reduced Bid expression attenuated neuronal death in a model of OGD in vitro and reduced brain damage in models of cerebral ischemia and brain trauma in vivo. ${ }^{21,31}$ 
a
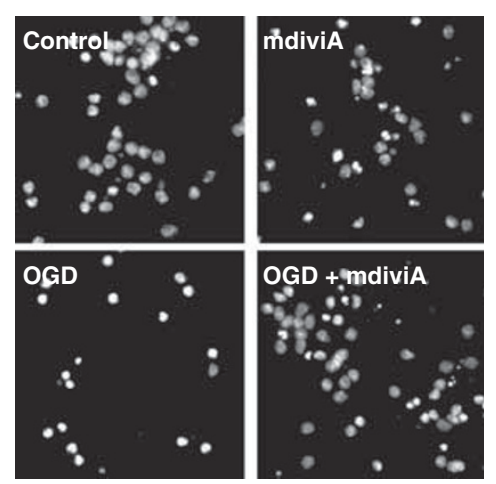

\section{b}

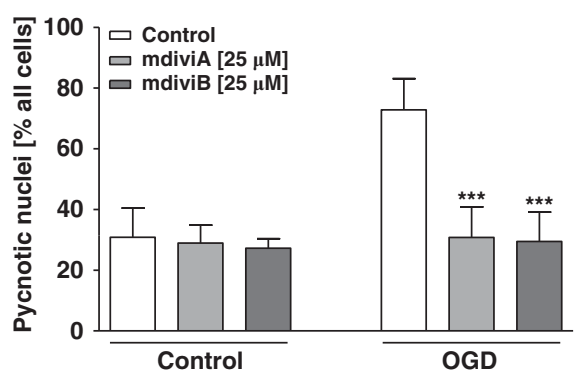

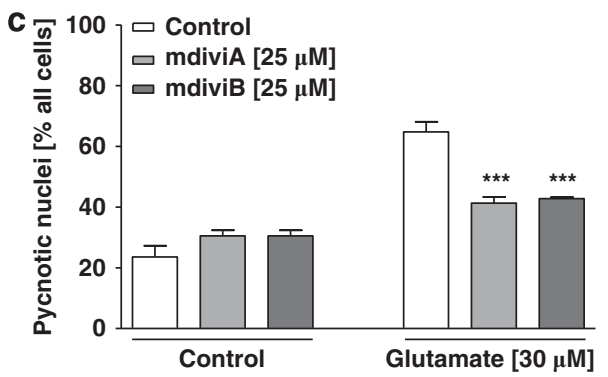
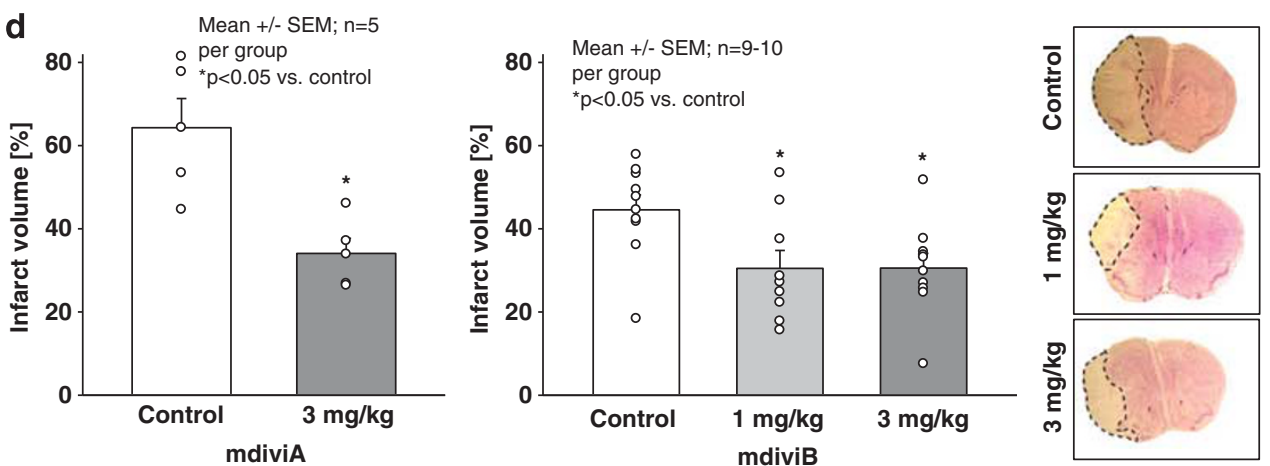

Figure 6 mdivi compounds protect primary neurons against glutamate-induced excitotoxicity and OGD in vitro and attenuate ischemic brain damage in vivo. Primary rat cortical neurons (DIV 9) were exposed $4 \mathrm{~h}$ to OGD. After $4 \mathrm{~h}$ of OGD, the $1 \times$ EBSS \pm glucose was replaced by NB + -medium containing glucose to all dishes and the cells were incubated for another $18-24 \mathrm{~h}$ in the regular oxygen-containing incubator. (a) Fluorescence microscope images ( $\times 40$ objective) of DAPI-stained embryonic cortical neurons were obtained after $18 \mathrm{~h}$ of exposure to OGD. (b) Counting of $\sim 500 \mathrm{DAPI}$-stained neurons with a total $n=5$ per group displaying pycnotic nuclei after $4 \mathrm{~h}$ of OGD revealed that $25 \mu \mathrm{M}$ Drp1 inhibitor mdiviA and B significantly protect neurons from hypoxic-hypoglycemic cell death. ${ }^{* \star} P<0.001$ versus untreated neurons subjected to OGD ( $n=5$, ANOVA, Scheffés-test). Cultures treated with $25 \mu \mathrm{M}$ mdiviA or B contained $>60 \%$ healthy nuclei, whereas all other OGD-treated groups show $75 \%$ pycnotic and/or fragmented nuclei, indicating apoptotic damage (OGD, right white column). The respective control cultures with $1 \times$ EBSS medium + glucose contained only very few apoptotic nuclei (Control, left white column). The experiments were repeated three times and the results are presented as mean \pm S.D. (c) Quantification of pycnotic nuclei in percentage of four independent experiments with a total $n=5$ per group. Primary rat cortical neurons (DIV 9) were treated for $18 \mathrm{~h}$ with glutamate ( $30 \mu \mathrm{M}$ ) and mdiviA and $\mathrm{B}(25 \mu \mathrm{M})$. After $18 \mathrm{~h}$, cells were fixed in $4 \% \mathrm{PFA}$ and stained with DAPI and $\sim 500$ nuclei were counted per treatment condition. MdiviA and B significantly reduced glutamateinduced cell death. ${ }^{* \star *} P<0.001$ versus glutamate-treated neurons ( $n=5$, ANOVA, Scheffé's-test). (d) Transient focal ischemia was induced for 45 min by a silicon-coated nylon filament that was introduced into the internal carotid artery to occlude the middle cerebral artery (MCA). Surgery was performed in deep isoflurane/ $\mathrm{N}_{2} \mathrm{O}$ anesthesia with controlled ventilation, and ischemia and reperfusion were verified by Laser-Doppler flowmetry. The infarct volume was calculated on the basis of the histomorphometric data from 12 to 15 consecutive sections ( $10 \mu \mathrm{M}, 500 \mu \mathrm{M}$ apart) obtained $24 \mathrm{~h}$ after onset of ischemia. MdiviA ( $3 \mathrm{mg} / \mathrm{kg})$ and mdiviB ( 1 and $3 \mathrm{mg} / \mathrm{kg})$ reduced the mean infarct volume by 34 and $30 \%$ compared with vehicle (DMSO) controls, respectively. The photomicrographs show representative pictures of cresyl-violet-stained brain slices from animals, which received vehicle or mdiviB ( 1 and $3 \mathrm{mg} / \mathrm{kg}$ ). The infarct area remained unstained and is highlighted by dashed lines

Recent studies showed that tBid and full-length Bid exert similar effects on mitochondria and AIF-dependent cell death in neurons. ${ }^{19,32}$ Mitochondrial translocation of full-length Bid was sufficient to induce Bax oligomerization and integration into the outer mitochondrial membrane in Hela cells ${ }^{33}$ and to induce mitochondrial dysfunction in neurons. ${ }^{32}$ Activated tBid induces rapid mitochondrial demise in caspase-dependent death receptor mediated pathways, while full-length Bidmediated caspase-independent cell death pathways, however with slower kinetics compared with tBid. ${ }^{23}$ In the present model system, activated full-length Bid and tBid may both contribute to the mitochondrial death pathways that are observed in HT-22 cells with a delay of 6-8 h after onset of glutamate exposure. 
Our data suggest an essential interplay of proapoptotic Bid with Drp1, the main regulator of mitochondrial fission. We found that tBid-induced loss of MOMP and mitochondrial fragmentation in a Drp1-dependent manner, since Drp1 inhibition and Drp1 siRNA prevented tBid-induced mitochondrial fission and cell death (Figure 5). Further, Bid siRNA prevented mitochondrial translocation of Drp1 to the mitochondria after glutamate exposure, suggesting an interaction between these proteins in mitochondrial damage and associated death pathways (Supplementary Figure 8e). A direct interaction between Bid/tBid and Drp1, however, could not be detected by immunoprecipitation (Supplementary Figure 9) and the collaborative activity of Drp1 and Bid in mitochondrial death pathways may thus be mediated through more complex mechanisms. For example, the interplay between Bid and Drp1 may involve other proapoptotic Bcl-2 proteins such as Bax. It was previously reported, that in addition to full-length Bid and truncated Bid additional proteins are required to trigger Bax oligomerization at the MOM sites. ${ }^{33-36}$ Consistent with previous reports, ${ }^{28}$ we found that Bax co-immunoprecipitated with Drp1 after glutamate toxicity, suggesting that in our model system, Bax and Drp1 cooperate to promote MOMP and mitochondrial fission (Supplementary Figure 9).

This conclusion is confirmed by results showing that Bid induces the oligomerization and insertion of Bax in the $\mathrm{MOM}^{33}$ and Bax was also found to co-localize with Drp1 at fission sites. ${ }^{28,37}$ Further, very recent findings demonstrated Drp1 recruitment to cardiolipin-rich membrane sites as a prerequisite for the formation of membrane hemifusion/fission structures and Bax oligomerization. ${ }^{11,28}$ Notably, Drp1-induced Bax oligomerization at mitochondrial fission sites also required the presence of $\mathrm{tBid}$, which further supports the conclusion that both, Drp1 and Bid concomitantly contribute to mitochondrial death pathways. ${ }^{11,29}$ Altogether, these findings and our data in neuronal cells suggest that Bid and oligomerized Bax accumulate at Drp1 recruiting sites in the MOM, which then become mitochondrial fission sites.

In damaged neurons, inhibition of Drp1 may serve to shift the observed mitochondrial fission back to a balance of fusion and fission thereby preventing neuronal dysfunction and death. Alternatively, the role of Drp1 in neuronal cell death may be independent of its role in mitochondrial fission. Bax oligomerization and subsequent release of proapoptotic factors to the cytosol were significantly delayed in cells expressing Drp1 mutants lacking membrane recruitment and hemifusion activity. ${ }^{11}$ This observation is in line with our present findings of Drp1 recruitment to mitochondria before demise of these organelles. Thus, in dying neurons, Bid- and Drp1-dependent mitochondrial fission may be regarded as a morphological hallmark initiating the process of MMP and mitochondrial death pathways.

In summary, the inhibition of Drp1 using the mdivi compounds represents a potential therapeutic strategy for both acute and chronic neurological diseases associated with glutamate toxicity and oxidative stress. This approach may prevent neuronal death pathways upstream of mitochondrial damage thereby providing persistent neuroprotective effects in a wide variety of different neurological diseases where programmed cell death causes dysfunction and death of neurons.

\section{Materials and Methods}

Cell culture and induction of neuronal cell death. HT-22 cells derived from immortalized hippocampal neurons were cultured in Dulbecco's modified Eagle medium (DMEM; Invitrogen, Karlsruhe, Germany) supplemented with $10 \%$ heatinactivated fetal calf serum, $100 \mathrm{U} / \mathrm{ml}$ penicillin, $100 \mu \mathrm{g} / \mathrm{ml}$ streptomycin and $2 \mathrm{mM}$ glutamine (PAA Laboratories $\mathrm{GmbH}$, Cölbe, Germany). Induction of apoptosis was performed $24 \mathrm{~h}$ after seeding of the cells. Growth medium was removed and replaced by medium containing inhibitors and glutamate ( 3 and $5 \mathrm{mM}$ ) for induction of apoptosis. Between 2 and $18 \mathrm{~h}$ later, cells were analyzed following standard procedures for flow cytometry, epifluorescence microscopy, protein or RNA analysis. It is important to note that sensitivity to glutamate toxicity varies in $\mathrm{HT}-22$ neurons depending on their density and passage number. Therefore, experiments were always performed at different glutamate concentrations with 3 and $5 \mathrm{mM}$, and representative results are mostly shown from at least three pooled independent experiments where the respective glutamate concentration induced at least $50 \%$ cell death as detected with the MTT assay.

Primary rat embryonic cortical neurons were cultured in neurobasal medium with $2 \%$ (v/v) B27 Supplementary, $2 \mathrm{mM}$ glutamine and $100 \mathrm{U} / \mathrm{ml}$ penicillin/streptomycin (Invitrogen, San Diego, CA, USA). Cultures contain $>95 \%$ neurons as routinely controlled by neuronal-specific nuclear protein (NeuN) immunostaining. ${ }^{31,38} \ln 6$ - to 7-day-old primary neurons, the culture medium was replaced immediately before glutamate treatment $(25 \mu \mathrm{M}, 24$ and $48 \mathrm{~h})$. Since cultures of primary neurons develop functional glutamate receptors after 6-8 days in culture, experimental treatments were performed with 8- to 10-day-old cultures in Earl's balanced salt solution ( $1 \times$ EBSS) medium (in mg/l: $6800 \mathrm{NaCl}, 400 \mathrm{KCl}, 264 \mathrm{CaCl}_{2} \times 2 \times \mathrm{H}_{2} \mathrm{O}$, $200 \mathrm{MgCl}_{2} \times 7 \times \mathrm{H}_{2} \mathrm{O}, 2200 \mathrm{NaHCO}_{3}, 140 \mathrm{NaH}_{2} \mathrm{PO}_{4} \times \mathrm{H}_{2} \mathrm{O}$ and 1000 glucose, $\mathrm{pH}$ 7.2). Apoptosis was induced by glutamate $(30 \mu \mathrm{M})$ in $1 \times$ EBSS and quantified $18-24 \mathrm{~h}$ later. Cells were treated with mdiviA or mdiviB $25 \mu \mathrm{M}$ in $1 \times$ EBSS. After $18-24 \mathrm{~h}$, the neurons were fixed with $4 \%$ paraformaldehyde (PFA) and stained with the fluorescent DNA-binding dye DAPI (4',6-diamidino-2-phenylindole dihydrochloride) (Sigma-Aldrich, Taufkirchen, Germany). Living and pyknotic cells were counted using a fluorescence microscope (DMI6000B, Leica, Wetzlar, Germany) without knowledge of the treatment history. For the quantification of apoptotic cells, 500 cells from five areas per cell culture dish were counted in total and experiments were performed at least three times with $n=5$ per treatment condition.

For OGD. Primary cortical neurons were seeded onto $35 \mathrm{~mm}$ culture dishes with $5 \times 10^{4} / \mathrm{cm}^{2}$ cells. At day 8 or 9 in culture, the dishes were separated into two groups, one for the OGD chamber and one as control group. First, all dishes were washed twice with glucose-free $1 \times$ EBSS supplemented with gentamycin $(5 \mathrm{mg} / \mathrm{l})$. For OGD, the cells were treated with mdiviA or mdiviB $25 \mu \mathrm{M}$ in $1 \times$ EBSS without glucose and transferred to the chamber containing $5 \%$ carbon dioxide $\left(\mathrm{CO}_{2}\right)$ and $95 \% \mathrm{~N}_{2}$ for $4 \mathrm{~h}$. The analog treatment in $1 \times$ EBSS containing glucose $(10 \mathrm{mM})$ or neurobasal was applied to the control cells in the regular oxygen-containing incubator. After $4 \mathrm{~h}$ of OGD, the $1 \times$ EBSS \pm glucose was replaced by NB +medium containing glucose to all dishes and the cells were incubated for another $18-24 \mathrm{~h}$ in the regular oxygen-containing incubator. For counting analysis of cell death, cells were fixed with 4\% PFA and stained with the fluorescent DNA-binding dye DAPI (Sigma-Aldrich). Living and pycnotic cells were counted using a fluorescence microscope (DMI6000B, Leica) without knowledge of the treatment history. For the quantification of apoptotic cells, 500 cells from five areas per cell culture dish were counted in total and experiments were performed at least three times with $n=5$ per treatment condition.

Plasmids and gene transfer. The mGFP vector, the IRES-tBid vector and control vectors were generated as described previously. ${ }^{5,19}$ For plasmid and siRNA transfections $7 \times 10^{4}$ HT-22 cells were seeded in 24-well plates. Standard growth medium was replaced with $900 \mu \mathrm{l}$ antibiotic-free growth medium. Lipofectamine 2000 (Invitrogen, Germany), IRES-tBid, mGFP vector or empty vector pcDNA 3.1 + were dissolved separately in Optimem I (Invitrogen, Germany). After 10 min of equilibration at room temperature, each DNA solution was combined with the respective volume of the Lipofectamine 2000 solution, mixed gently, and allowed to form plasmid liposomes for further $20 \mathrm{~min}$ at room temperature. The transfection mixture was added to the antibiotic-free cell culture medium to a final concentration of $1 \mu \mathrm{g} \mathrm{DNA}$ and $1.5 \mu \mathrm{l} / \mathrm{ml}$ Lipofectamine $2000 \mathrm{in} \mathrm{HT}-22$ cells. Controls were treated with $100 \mu \mathrm{l} / \mathrm{ml}$ Optimem only, and vehicle controls with $1.5 \mu \mathrm{l} / \mathrm{ml}$ Lipofectamine 2000.

Inhibition of Drp1. Drp1 inhibition was achieved by using highly specific mdivi compounds A, B, C, E and G in HT-22 cells at concentrations of 50 and $75 \mu \mathrm{M}$ and 
at concentrations of $25 \mu \mathrm{M}$ in primary neurons. ${ }^{17}$ Notably, higher concentrations of the mdivi compounds exerted toxic effects in the respective cell cultures.

Drp1 silencing. Lipofectamine 2000 (Invitrogen, Germany) and Drp1 siRNA (On-target Plus siRNA Pool (four different sequences), Dharmacon, Thermo Scientific, Bonn, Germany) or non-functional mutant RNA (5'-AAG AGA AAA AGC GAA GAG CCA-3'; Dharmacon, Thermo Scientific) were dissolved separately in Optimem I (Invitrogen, Germany). After $10 \mathrm{~min}$ of equilibration at room temperature, each RNA solution was combined with the respective volume of the Lipofectamine 2000 solution, mixed gently and allowed to form siRNA liposomes for $20 \mathrm{~min}$ at room temperature. The transfection mixture was added to the antibiotic-free cell culture medium to a final concentration of 60 and $80 \mathrm{nM} \mathrm{RNA}$ and $2 \mu \mathrm{l} / \mathrm{ml}$ Lipofectamine in HT-22 cells. Controls were treated with $100 \mu \mathrm{l} / \mathrm{ml}$ Optimem only and vehicle controls with $2 \mu \mathrm{l} / \mathrm{ml}$ Lipofectamine 2000.

Immunostaining and confocal laser scanning fluorescence microscopy. For detection of mitochondrial morphology changes and Drp1 translocation during cell death, $\mathrm{HT}-22$ cells were transfected with the mGFP plasmid with Lipofectamine 2000. Twenty-four hours after transfection, HT-22 cells were seeded in ibidi $\mu$-slide eight-well plates (Ibidi GmbH, Munich, Germany) at a density of $1.7 \times 10^{4} \mathrm{cells} /$ well for end point analysis on a fluorescence microscope (DMI6000B, Leica). Alternatively, mitochondria were visualized by MitoTracker Green/DeepRed according to the manufacturer's protocol (Invitrogen, Germany). Microscopic pictures were taken after fixation of the cells with 4\% PFA 4-18 h after onset of treatment, followed by Drp1 immunostaining using a specific Drp1 antibody (BD Transduction Laboratories, Heidelberg, Germany) and DAPI counterstaining of the nuclei. Images were acquired using a fluorescent confocal laser scanning microscope (LSM 510, Carl Zeiss, Jena, Germany) equipped with an UV, an argon, and a Helium/Neon laser delivering light at 364,488 and $543 \mathrm{~nm}$, respectively. Light was collected $\times 631.4 \mathrm{NA}$, oil immersion objective. DAPI fluorescence was excited at $364 \mathrm{~nm}$ and emission was achieved by using the $385-\mathrm{nm}$ long pass filter. Mitotracker Green and mGFP were excited at 488 and $543 \mathrm{~nm}$ and emissions were detected using $505-530 \mathrm{~nm}$ band pass filter (green), and Mitotracker DeepRed was excited at $620 \mathrm{~nm}$ band pass filter and emissions were detected using $690 \mathrm{~nm}$ long pass filter (red). Fluorescence of oregon green was excited at a wavelength of $488 \mathrm{~nm}$ and emission was detected using a $505-\mathrm{nm}$ long pass filter. For digital imaging, the software LSM Image Browser 4.2.0 (Carl Zeiss) was used.

Evaluation of mitochondrial morphology. HT-22 cells were transfected with mGFP as described before or alternatively, mitochondria were visualized by MitoTracker Green/DeepRed according to the manufacturer's protocol (Invitrogen, Germany). After $24 \mathrm{~h}$, the cells were reseeded on eight-well-ibidi-slides (ibidi) and treated for $18 \mathrm{~h}$. Microscopic pictures were taken after fixation with $4 \%$ PFA and DAPI counterstaining of the nuclei $18 \mathrm{~h}$ after onset of treatment. Counting of four different types of mitochondrial morphology states was performed for quantification in at least four independent experiments with 500 cells per condition in $n=2$ wells by an investigator blinded towards the treatment of the cells. Three categories of mitochondrial morphology states were defined as follows: healthy cells display category 1 , mitochondria form elongated tubular network. These mitochondria are equally distributed throughout the cytosol. Cells containing large round mitochondria distributed throughout the cytosol are defined as category 2. These cells do not show apoptotic features, for example, shrunken nuclei, and are not detached. In contrast, damaged and dying cells contain smaller round heterogeneous mitochondria located close to the nucleus. These mitochondria are defined as category 3. For statistical analysis, the experiments were repeated at least three times. For automated analyses of mitochondrial morphology changes, mitochondrial length was also calculated by ImageJ software (NIH, Bethesda, MD, USA) from at least six independent pictures per experimental condition. ${ }^{5}$ Imaging of mitochondrial morphology was performed using a DMI6000B fluorescence microscope, equipped with a DCF360FX-camera (Leica). Mitotracker Green and mGFP were excited at 488 and $543 \mathrm{~nm}$ and emissions were detected using $505-530 \mathrm{~nm}$ band pass filter (green), and Mitotracker DeepRed was excited at $620 \mathrm{~nm}$ band pass filter and emissions were detected using $690 \mathrm{~nm}$ long pass filter (red).

RT-PCR. Total RNA was extracted (Nucleospin RNAll kit, Macherey-Nagel, Düren, Germany) and One-step RT-PCR was performed with Superscriptlll One Step RT-PCR (Invitrogen, Germany). Primers for Drp1 and GAPDH were used as follows: Drp1 forward primer, 5'-ACAGGAGAAGAAAATGGAGTTTGAAGCAG-3', Drp1 reverse primer, 5'-AACAAATCCTAGCACCACGCAT-3', GAPDH forward primer 5'-CGTCTTCACCACCATGGAGAAGGC- $3^{\prime}$, and GAPDH reverse primer, $5^{\prime}$-AAGGCCATGCCAGTGAGCTTCCC- $3^{\prime}$. PCR for GAPDH and Drp1 was performed after $\mathrm{CDNA}$-synthesis at $60^{\circ} \mathrm{C}$ for $30 \mathrm{~min}$ and a denaturation at $95^{\circ} \mathrm{C}$ for $2 \mathrm{~min}$. PCR amplification was performed by 26 cycles, each with a denaturation for $30 \mathrm{~s}$ at $95^{\circ} \mathrm{C}$, annealing for $1 \mathrm{~min}$ at $57^{\circ} \mathrm{C}$ and elongation for $2 \mathrm{~min}$ at $70^{\circ} \mathrm{C}$. The final extension of the PCR products was performed at $70^{\circ} \mathrm{C}$ for $10 \mathrm{~min}$. RT-PCR products were visualized by a Bio-Rad gel detection system (ChemiDoc XRS BioRad, Karlsruhe, Germany) under UV illumination after electrophoresis on a 1.5\% agarose gel containing SybrGold (Invitrogen, Germany).

Evaluation of cell viability and apoptosis. For morphological analysis of cell viability, transmission light microscopy of living HT-22 cells growing as monolayers was performed using an Axiovert 200 microscope (Carl Zeiss) equipped with a Lumenera Infinity 2 digital camera (Lumenera Corporation, Ottawa, Canada). Light was collected through a $\times 100.25$ NA objective (Carl Zeiss), and images were captured using phase contrast. Digital image recording and image analysis were performed with the INFINITY ANALYZE software (Lumenera Corporation). Quantification of cell viability in HT-22 cells was performed in 96-well plates with 7000 cells/well by MTT reduction at $0.25 \mathrm{mg} / \mathrm{ml}$ for $1 \mathrm{~h}$. The reaction was terminated by adding DMSO after freezing the plate without media at $-80^{\circ} \mathrm{C}$ for at least $1 \mathrm{~h}$ and absorbance was then determined at $590 \mathrm{~nm}$ versus $630 \mathrm{~nm}$ (Fluostar OPTIMA, BMG Labtech, Offenburg, Germany). The data are normalized to DMSO control when mdivi compounds were used in the experiment. In the case of siRNA applications, the presented cell viability data are normalized to the vehicle control Lipofectamine 2000 or Lipofectamine RNAiMax (Invitrogen, Germany). The controls were set to $100 \%$ cell viability, since absolute numbers may vary between experiments depending on cell density and MTT signal variations between independent experiments. For statistical analysis, the experiments were repeated at least three times with an $n=8$ per treatment condition.

For end point analysis of total ATP levels, the ViaLight HS Kit (Lonza, Cologne, Germany) was used following the manufacturer's instructions. HT-22 cells were seeded in white 96-well plates (Greiner, Frickenhausen, Germany) for luminescence measurements. Twenty-four hours after seeding, the cells were damaged with glutamate 3 and $5 \mathrm{mM}$ and treated with Drp1 inhibitor mdiviA $(75 \mu \mathrm{M})$. ATP levels were detected $0-14 \mathrm{~h}$ after the onset of glutamate exposure by detection of luminescence. The ATP release levels were expressed as percentage of luminescence levels in treated control cells (100\% ATP release). For statistical analysis, the experiments were repeated at least three times with an $n=8$ per treatment condition.

Apoptotic cell death was detected by Annexin-V/propidium iodide staining and subsequent flow cytometry analysis. Cells from three-wells per condition were harvested $14 \mathrm{~h}$ after glutamate treatment by using Trypsin/EDTA $(1 \times$ TE; SigmaAldrich), washed once in $1 \times$ PBS and stained according to the manufacturer's protocol (Annexin-V-FITC Detection Kit, PromoKine, Promocell, Heidelberg, Germany). Apoptotic and necrotic cells were determined using FACScan (BD Bioscience, Heidelberg, Germany). Annexin-V-FITC was excited at $488 \mathrm{~nm}$ and emission was detected through a $530 \pm 40 \mathrm{~nm}$ band pass filter. Propidium iodide was excited at $488 \mathrm{~nm}$ and fluorescence emission was detected using a $680 \pm 30 \mathrm{~nm}$ band pass filter. To exclude cell debris and doublets, cells were appropriately gated by forward versus side scatter and pulse width, and $1 \times 10^{4}$ gated events per sample were collected. Surviving cells did not show any staining whereas Annexin- $V$ staining indicated apoptosis and cells positive for both Annexin$\mathrm{V}$ and propidium iodide were regarded necrotic. For statistical analysis, the experiments were repeated at least three times.

DAPI staining. At different time points after the onset of the different treatment conditions, cultured primary neurons were fixed for $15 \mathrm{~min}$ in $1 \mathrm{ml}$ of a $1 \times$ PBS solution containing $4 \%$ PFA. The fixed primary neurons were stained for $15 \mathrm{~min}$ in $35 \mathrm{~mm}$ dishes with the fluorescent DNA-binding dye DAPI or Hoechst 33342 $(1 \mu \mathrm{g} / \mathrm{ml}$ in $1 \times$ PBS$)$. After removing the fluorescent dyes, cells were washed twice with $1 \times$ PBS and kept in $1 \mathrm{ml} 1 \times$ PBS. Stained nuclei were visualized under epifluorescence illumination (DMI6000B, Leica) with A4 (340 nm excitation and $510 \mathrm{~nm}$ barrier filter) using a $\times 40$ objective. Neurons with condensed and fragmented nuclei were considered apoptotic. For the quantification of apoptotic cells, 500 cells from five areas per cell culture dish were counted in total and experiments were performed at least three times with $n=5$ per treatment condition without knowledge of the treatment history. For statistical analysis, the experiments were repeated at least three times. 
Detection of cellular lipid peroxidation. For detection of cellular lipid peroxidation, HT-22 cells were seeded out in 24 -well plates with $7 \times 10^{5}$ cells $/$ well. After respective treatments, the cells were loaded with $2 \mu \mathrm{M}$ BODIPY 581/591 C11 for $60 \mathrm{~min}$ in $1 \mathrm{ml}$ DMEM per well. Cells were then harvested with standard $1 \times \mathrm{TE}$ in $1 \times$ PBS with $100 \mu /$ well. Trypsin incubation was stopped by adding $1 \mathrm{ml}$ serum containing medium to each well. Cells were centrifuged at $1.000 \times g$ for $5 \mathrm{~min}$ at room temperature, washed with $1 \times$ PBS and resuspended in $1 \mathrm{ml} 1 \times$ PBS. Detection of lipid peroxidation was performed by flow cytometry on a FACScan (BD Bioscience) by using $488 \mathrm{~nm}$ UV line argon laser for excitation and lipid peroxidation emission was recorded on channels FL1 at $530 \mathrm{~nm}$ (green) and FL2 at $585 \mathrm{~nm}$ (red). Data were collected from at least 20000 cells. To exclude cell debris and doublets, cells were appropriately gated by forward versus side scatter and pulse width, and $2 \times 10^{4}$ gated events per sample were collected from three to four independent samples per treatment condition.

Analysis of MMP. MMP of HT-22 neurons was determined by $5,5^{\prime}, 6$, $6^{\prime}$-tetrachloro-1, $1^{\prime}, 3,3^{\prime}$-tetraethylbenzimidazolylcarbocyanine iodide (JC-1) reduction. HT-22 neurons were stained with JC-1 (Mitoprobe, Invitrogen, Germany) according to the manufacturer's protocol and analyzed by subsequen flow cytometry or epifluorescence microscopy. After glutamate treatment (12 h), JC1 was added to each well of the different treatment conditions to a final concentration of $2 \mu \mathrm{M}$ for $30 \mathrm{~min}$. Living-control cells received vehicle (DMSO, $0.1 \%$ final concentration) and damage-control cells were treated with carbonyl cyanide $\mathrm{m}$ chlorophenylhydrazone (CCCP) 15 min before staining to induce mitochondrial membrane depolarization. Detached cells in the medium were collected and combined with the attached cells harvested with standard $1 \times$ TE in $1 \times$ PBS. Trypsin incubation was stopped by adding $1 \mathrm{ml}$ serum containing medium to each well. Cells were centrifuged at $1000 \times g$ for $5 \mathrm{~min}$ at room temperature, washed once with $1 \times$ PBS, and kept in $0.5 \mathrm{ml} 1 \times$ PBS on ice until analysis of JC-1 fluorescence using a FACScan (BD Bioscience). JC-1 green fluorescence indicating mitochondrial uptake of the dye was excited at $488 \mathrm{~nm}$ and emission was detected using a $530 \pm 40 \mathrm{~nm}$ band pass filter. JC-1 red fluorescence indicating intact MMP was excited at $488 \mathrm{~nm}$ and emission was detected using a $613 \pm 20 \mathrm{~nm}$ band pass filter. To exclude cell debris and doublets, cells were appropriately gated by forward versus side scatter and pulse width, and $1 \times 10^{4}$ gated events per sample were collected from three to four independent samples per treatment condition and the experiments were repeated at least three times.

Real-time measurements with xCelligence. The xCelligence System (Roche, Penzberg, Germany) monitors cellular events in real time by measuring the electrical impedance between micro-electrodes integrated into the bottom of custom made tissue culture plate (E-Plates). Since cells have a very high electrical resistance, the more cells are attached to the bottom of the E-Plate, the higher the electrical impedance will be. Thus, the electrical impedance, which is displayed as cell index $(\mathrm{Cl})$, can be used to monitor cell viability, number, morphology and adhesion in a large number of tissue culture wells simultaneously, at any given frequency, and over any desired period of time without removing the plate from the incubator. HT-22 cells were seeded in a density of $4000-5000$ cells/well in 96-well E-plates (Roche). Twenty-four hours after seeding, cells were incubated with vehicle, mdivi compound $\mathrm{A}(75 \mu \mathrm{M})$, glutamate ( 3 and $5 \mathrm{mM}$ ) or glutamate + mdivi compound $\mathrm{A}(75 \mu \mathrm{M})$.

Cerebral ischemia. Male C57BL/6 mice (body weight, 18-22 g; Charles River Laboratories, Wilmington, MA, USA) were subjected to transient MCAO as described previously. ${ }^{21,39}$ The Drp1 inhibitors mdiviA and B were injected i.p. at doses of 1 and $3 \mathrm{mg} / \mathrm{kg}$ body weight before the induction of MCAo. Surgery was performed in isoflurane/ $\mathrm{N}_{2} \mathrm{O}$ anesthesia ( $1.5 \%$ isoflurane, $68.5 \% \mathrm{~N}_{2} \mathrm{O}, 30 \% \mathrm{O}_{2}$ ). A silicone-coated nylon monofilament was inserted into the internal carotid artery and gently pushed forward until blood flow in the MCA territory decreased to $<20 \%$ of baseline as determined by Laser-Doppler fluxmetry. Twenty-four hours after MCAo, animals were perfused and fixed in 4\% PFA for $24 \mathrm{~h}$. Thereafter, the brains were removed, post-fixed in 4\% PFA overnight, dehydrated and embedded in paraffin. In all, $12-15$ sections ( $10 \mu \mathrm{M}, 500 \mu \mathrm{M}$ apart), including the middle cerebral artery territory, were stained with cresyl-violet for quantification of infarct area or evaluation of ischemic cell death, respectively. For each experimental group, five animals were used in the experiments with mdiviA, and 9-10 animals per group were used in the experiments with mdiviB. Statistical analysis was performed using the Mann-Whitney U-test. Physiological parameters and animal temperature were obtained as described previously. ${ }^{40}$ The left external carotid artery was cannulated for blood sampling, monitoring of blood gases, $\mathrm{pH}$, electrolyte status and mean arterial blood pressure (MABP) during isoflurane/ $\mathrm{N}_{2} \mathrm{O}$ anesthesia. Laser-Doppler flowmetry (LDF; MBF3D, Moor Instruments) was carried out to monitor local cortical blood flow (LCBF) of cortical areas supplied by the MCA. For the placement of the LDF probe, a burr hole ( $1 \mathrm{~mm}$ diameter) was drilled $5 \mathrm{~mm}$ lateral and $1 \mathrm{~mm}$ posterior to the bregma without injury of the dura mater. LCBF was continuously measured ( $2 \mathrm{~Hz}$ sampling rate) starting before the onset of ischemia and lasting as long as $1 \mathrm{~h}$ after reperfusion.

Statistical analysis. All data are given as mean values \pm standard deviation (S.D.). For statistical comparison between two groups, Mann-Whitney U-test was used. Multiple comparisons were performed by analysis of variance (ANOVA) followed by Scheffé's post hoc test or Bonferroni-test, as indicated. Calculations were performed with the Winstat standard statistical software package (R Fitch Software, Bad Krozingen, Germany).

\section{Conflict of Interest}

The authors declare no conflict of interest.

Acknowledgements. We thank Emma Esser for careful editing of the manuscript. Parts of this project were supported by a grant of the Michael J Fox Foundation to Carsten Culmsee.

1. Chan DC. Mitochondria: dynamic organelles in disease, aging, and development. Cell 2006; 125: 1241-1252.

2. Benard G, Bellance N, James D, Parrone P, Fernandez $H$, Letellier $T$ et al. Mitochondrial bioenergetics and structural network organization. J Cell Sci 2007; 120: 838-848.

3. Chen $\mathrm{H}$, McCaffery JM, Chan DC. Mitochondrial fusion protects against neurodegeneration in the cerebellum. Cell 2007; 130: 548-562.

4. Jezek P, Plecita-Hlavata L. Mitochondrial reticulum network dynamics in relation to oxidative stress, redox regulation, and hypoxia. Int J Biochem Cell Biol 2009; 41: 1790-1804.

5. Grohm J, Plesnila N, Culmsee C. Bid mediates fission, membrane permeabilization and peri-nuclear accumulation of mitochondria as a prerequisite for oxidative neuronal cell death. Brain Behav Immun 2009; 24: 831-838.

6. Westermann B. Mitochondrial fusion and fission in cell life and death. Nat Rev Mol Cell Biol 2010; 11: 872-884

7. Reddy PH, Reddy TP, Manczak M, Calkins MJ, Shirendeb U, Mao P. Dynamin-related protein 1 and mitochondrial fragmentation in neurodegenerative diseases. Brain Res Rev 2010; 67: 103-118.

8. Knott AB, Perkins G, Schwarzenbacher R, Bossy-Wetzel E. Mitochondrial fragmentation in neurodegeneration. Nat Rev Neurosci 2008; 9: 505-518.

9. Lackner LL, Nunnari JM. The molecular mechanism and cellular functions of mitochondrial division. Biochim Biophys Acta 2008; 1792: 1138-1144.

10. Arnoult D, Rismanchi N, Grodet A, Roberts RG, Seeburg DP, Estaquier J et al. Bax/Bakdependent release of DDP/TIMM8a promotes Drp1-mediated mitochondrial fission and mitoptosis during programmed cell death. Curr Biol 2005; 15: 2112-2118.

11. Montessuit S, Somasekharan SP, Terrones O, Lucken-Ardjomande S, Herzig S, Schwarzenbacher $\mathrm{R}$ et al. Membrane remodeling induced by the dynamin-related protein Drp1 stimulates Bax oligomerization. Cell 2010; 142: 889-901.

12. Frank S, Gaume B, Bergmann-Leitner ES, Leitner WW, Robert EG, Catez F et al. The role of dynamin-related protein 1, a mediator of mitochondrial fission, in apoptosis. Dev Cell 2001; 1: 515-525.

13. Lackner LL, Nunnari J. Small molecule inhibitors of mitochondrial division: tools that translate basic biological research into medicine. Chem Biol 2010; 17: 578-583.

14. Sagara Y, Dargusch R, Chambers D, Davis J, Schubert D, Maher P. Cellular mechanisms of resistance to chronic oxidative stress. Free Radic Biol Med 1998; 24: 1375-1389.

15. Burdo J, Dargusch R, Schubert D. Distribution of the cystine/glutamate antiporter system xc- in the brain, kidney, and duodenum. J Histochem Cytochem 2006; 54: 549-557.

16. Tobaben S, Grohm J, Seiler A, Conrad M, Plesnila N, Culmsee C. Bid-mediated mitochondrial damage is a key mechanism in glutamate-induced oxidative stress and AIFdependent cell death in immortalized HT-22 hippocampal neurons. Cell Death Differ 2011; 18: $282-292$

17. Cassidy-Stone A, Chipuk JE, Ingerman E, Song C, Yoo C, Kuwana T et al. Chemical inhibition of the mitochondrial division dynamin reveals its role in Bax/Bak-dependent mitochondrial outer membrane permeabilization. Dev Cell 2008; 14: 193-204.

18. Uo T, Dworzak J, Kinoshita C, Inman DM, Kinoshita Y, Horner PJ et al. Drp1 levels constitutively regulate mitochondrial dynamics and cell survival in cortical neurons. Exp Neurol 2009; 218: 274-285.

19. Landshamer S, Hoehn M, Barth N, Duvezin-Caubet S, Schwake G, Tobaben S et al. Bidinduced release of AIF from mitochondria causes immediate neuronal cell death. Cell Death Differ 2008; 15: 1553-1563. 
20. Culmsee C, Plesnila N. Targeting Bid to prevent programmed cell death in neurons, Biochem Soc Trans 2006; 34: 1334-1340.

21. Plesnila N, Zinkel S, Le DA, min-Hanjani S, Wu Y, Qiu J et al. BID mediates neuronal cell death after oxygen/glucose deprivation and focal cerebral ischemia. Proc Natl Acad Sci USA 2001; 98: 15318-15323.

22. Song G, Chen GG, Hu T, Lai PB. Bid stands at the crossroad of stress-response pathways. Curr Cancer Drug Targets 2010; 10: 584-592.

23. Ward MW, Rehm M, Duessmann H, Kacmar S, Concannon CG, Prehn JH. Real time single cell analysis of Bid cleavage and Bid translocation during caspase-dependent and neuronal caspase-independent apoptosis. J Biol Chem 2006; 281: 5837-5844.

24. Brooks $C$, Wei Q, Cho SG, Dong Z. Regulation of mitochondrial dynamics in acute kidney injury in cell culture and rodent models. J Clin Invest 2009; 119: 1275-1285.

25. Ong SB, Subrayan S, Lim SY, Yellon DM, Davidson SM, Hausenloy DJ. Inhibiting mitochondrial fission protects the heart against ischemia/reperfusion injury. Circulation 2010; 121: 2012-2022.

26. Park SW, Kim KY, Lindsey JD, Dai Y, Heo H, Nguyen DH et al. A selective inhibitor of drp1, mdivi-1, increases retinal ganglion cell survival in acute ischemic mouse retina. Invest Ophthalmol Vis Sci 2011; 52: 2837-2843.

27. Song W, Chen J, Petrilli A, Liot G, Klinglmayr E, Zhou Y et al. Mutant huntingtin binds the mitochondrial fission GTPase dynamin-related protein-1 and increases its enzymatic activity. Nat Med 2011; 17: 377-382.

28. Karbowski M, Norris KL, Cleland MM, Jeong SY, Youle RJ. Role of Bax and Bak in mitochondrial morphogenesis. Nature 2006; 443: 658-662.

29. Landes T, Martinou JC. Mitochondrial outer membrane permeabilization during apoptosis: the role of mitochondrial fission. Biochim Biophys Acta 2011; 1813: 540-545.

30. Becattini B, Culmsee C, Leone M, Zhai D, Zhang X, Crowell KJ et al. Structure-activity relationships by interligand NOE-based design and synthesis of antiapoptotic compounds targeting Bid. Proc Natl Acad Sci USA 2006; 103: 12602-12606.
31. Culmsee C, Zhu C, Landshamer S, Becattini B, Wagner E, Pellecchia M et al. Apoptosisinducing factor triggered by poly(ADP-ribose) polymerase and Bid mediates neuronal cell death after oxygen-glucose deprivation and focal cerebral ischemia. J Neurosci 2005; 25: 10262-10272.

32. Konig HG, Rehm M, Gudorf D, Krajewski S, Gross A, Ward MW et al. Full length Bid is sufficient to induce apoptosis of cultured rat hippocampal neurons. BMC Cell Biol 2007; 8: 7.

33. Eskes R, Desagher S, Antonsson B, Martinou JC. Bid induces the oligomerization and insertion of Bax into the outer mitochondrial membrane. Mol Cell Biol 2000; 20: 929-935.

34. Lovell JF, Billen LP, Bindner S, Shamas-Din A, Fradin C, Leber B et al. Membrane binding by tBid initiates an ordered series of events culminating in membrane permeabilization by Bax. Cell 2008; 135: 1074-1084.

35. Lucken-Ardjomande S, Montessuit S, Martinou JC. Contributions to Bax insertion and oligomerization of lipids of the mitochondrial outer membrane. Cell Death Differ 2008; 15: 929-937.

36. Terrones O, Etxebarria A, Landajuela A, Landeta O, Antonsson B, Basanez G. BIM and tBID are not mechanistically equivalent when assisting $B A X$ to permeabilize bilayer membranes. J Biol Chem 2008; 283: 7790-7803.

37. Wasiak S, Zunino R, McBride HM. Bax/Bak promote sumoylation of DRP1 and its stable association with mitochondria during apoptotic cell death. J Cell Biol 2007; 177: 439-450.

38. Culmsee C, Gerling N, Lehmann M, Nikolova-Karakashian M, Prehn JH, Mattson MP et al. Nerve growth factor survival signaling in cultured hippocampal neurons is mediated through TrkA and requires the common neurotrophin receptor P75. Neuroscience 2002; 115: $1089-1108$.

39. Plesnila N, Zhu C, Culmsee C, Groger M, Moskowitz MA, Blomgren K. Nuclear translocation of apoptosis-inducing factor after focal cerebral ischemia. J Cereb Blood Flow Metab 2004; 24: 458-466.

40. Culmsee C, Junker V, Kremers W, Thal S, Plesnila N, Krieglstein J. Combination therapy in ischemic stroke: synergistic neuroprotective effects of memantine and clenbuterol. Stroke 2004; 35: 1197-1202.

Supplementary Information accompanies the paper on Cell Death and Differentiation website (http://www.nature.com/cdd) 